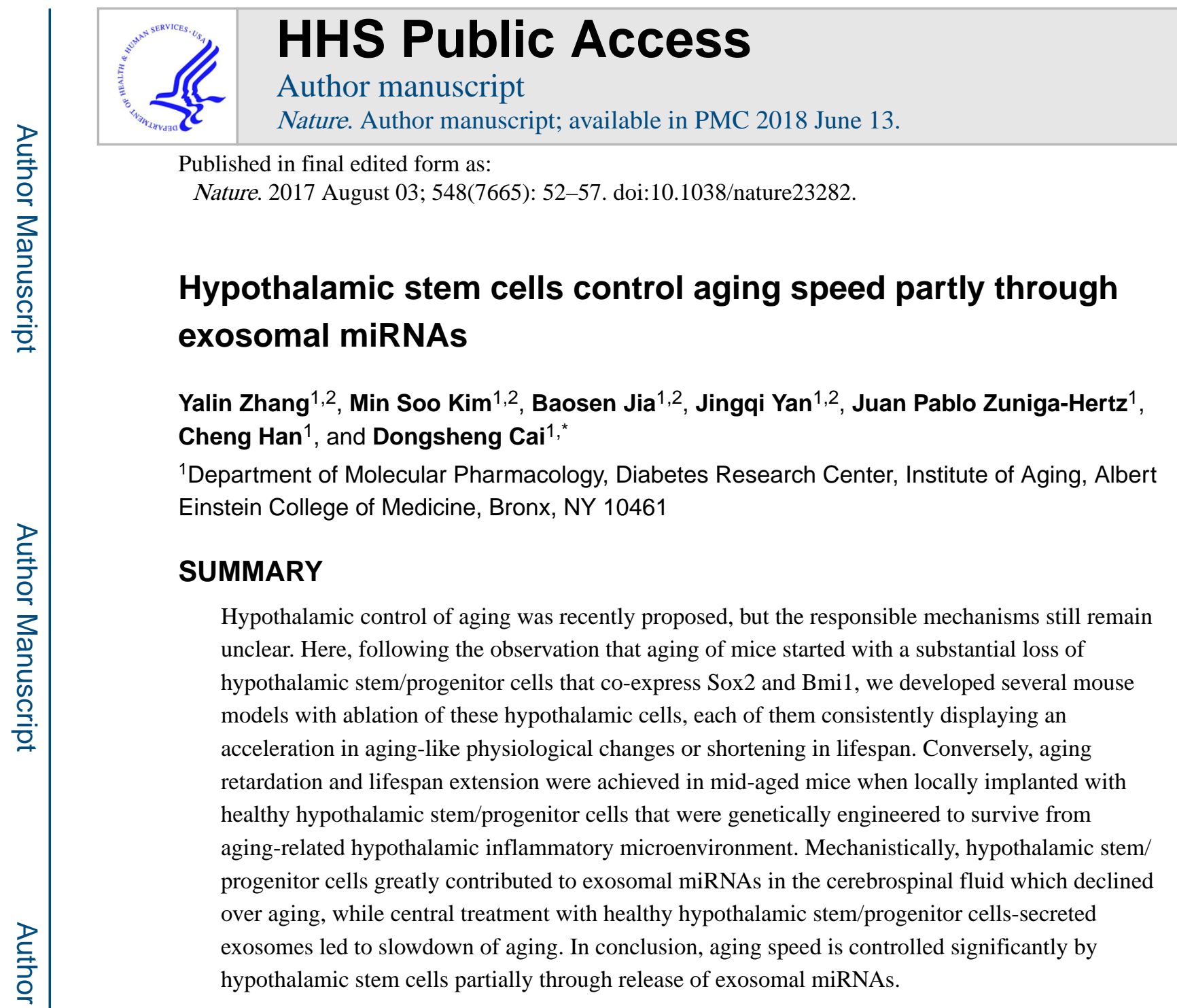

\title{
Keywords
}

Aging; hypothalamus; neural stem cell; miRNA; exosome

\begin{abstract}
While the nervous system clearly plays a role in aging ${ }^{1-6}$, and recent research has demonstrated that the hypothalamus is particularly important ${ }^{5-8}$, the responsible mechanism is still unknown. In neuroscience research, it has been appreciated that adult neural stem/ progenitor cells (NSC) reside in a few brain regions to mediate local neurogenesis and therefore several aspects of brain functioning ${ }^{9-13}$. Decline in adult neurogenesis is known to
\end{abstract}

\footnotetext{
Users may view, print, copy, and download text and data-mine the content in such documents, for the purposes of academic research, subject always to the full Conditions of use: http://www.nature.com/authors/editorial_policies/license.html\#terms

Correspondence should be addressed to D.C. (dongsheng.cai@einstein.yu.edu), Phone: 718-430-2426, Fax: 718-430-8922,

dongsheng.cai@einstein.yu.edu.

2 These authors equally contributed to this work

COMPETING INTERESTS STATEMENT

The authors state that they have no competing financial interests.

Author contribution

Y.Z. M.K. J.Y. and C.H. performed hypothalamic injections and cell implantation; Y.Z. performed immunostaining, cloning, virus production and CSF sampling; M.K. performed behavioral experiments and exosome treatment; B.J. performed cell culture, exosome and miRNA characterization; J.Y. performed lifespan follow-up and initial behavioral and miRNA analysis; J.Z. performed cell culture and imaging; D.C. conceived the hypothesis, designed the study and wrote the paper.
} 
correlate with advent of aging-associated disorders ${ }^{10,14-18}$. Such adult neurogenesis has mostly been studied for the hippocampus and the subventricular zone of lateral ventricle in the brain ${ }^{9-13}$. Of interest, as noted in recent literature ${ }^{19-21}$, adult NSC are significantly present in the hypothalamus, in particular the mediobasal hypothalamic region (MBH) which is crucial for the neuroendocrine regulation of whole body's physiological homeostasis. Here, supported by our recent work showing that the hypothalamus has a programmatic role in causing systemic aging ${ }^{5}$, we investigated if these hypothalamic NSC might be mechanistically responsible for this process.

\section{Aging-associated hypothalamic NSC loss}

Sox 2 is a nuclear transcription factor that is strongly expressed in NSC and has often been used to label $\mathrm{NSC}^{22}$. We recently found in adult mice that Sox 2 is expressed in a population of cells in the MBH and especially the hypothalamic third-ventricle (3V) wall within the $\mathrm{MBH}^{19}$. To gain an insight into their relevance to aging, we comparatively examined these hypothalamic cells during aging progression. Together with Sox2, we analyzed Polycomb complex protein Bmil (B lymphoma Mo-MLV insertion region 1 homolog) - a nuclear protein which is critical for self-renewal of NSC and hematopoietic stem cells ${ }^{23}$. As shown in Fig. 1a, cells co-expressing Sox2 and Bmi1 were found densely present in hypothalamic $3 \mathrm{~V}$ wall while sporadically in the $\mathrm{MBH}$ parenchyma of young mice, but these cells gradually diminished with the increase of age, starting from the bottom MBH portion of hypothalamic $3 \mathrm{~V}$ wall at mid-aged mice (11-16 months old) and becoming almost entirely lost in aged mice (22 months and older). We also examined nestin, which, although not NSC-specific, is known to be strongly expressed in NSC and their offspring progenitors ${ }^{20,24,25}$. To focus on the hypothalamic $3 \mathrm{~V}$ wall, we confirmed that nestin-positive cells in this region greatly declined with aging (Fig. 1b). In addition, since Musashi1 and CxCR4 were also reported as NSC biomarkers ${ }^{24,26}$, we examined both of them and found that cells positive for Musashi1 and $\mathrm{CxCR} 4$ in hypothalamic 3V wall dramatically declined during aging. Moreover, through BrdU tracking we confirmed that BrdU-positive cells were present in the hypothalamic $3 \mathrm{~V}$ wall of young mice but were barely detected in this region of mid-aged mice. Overall, the time course of age-dependent loss in hypothalamic NSC well correlated with aging-related physiological declines (Extended Data Fig. 1), further provoking us to investigate if the loss of these cells could be causally important for aging.

\section{Aging acceleration due to hypothalamic NSC loss}

As the hypothalamic $3 \mathrm{~V}$ wall is the site which sensitively undergoes loss of NSC, we mimicked this change through experimentally ablating NSC in this region. To do so, we adopted the approach of viral injection in hypothalamic $3 \mathrm{~V}$, especially because injected lentiviruses infected the $3 \mathrm{~V}$ surface which contains NSC but barely penetrated the wall to infect the parenchyma (Extended Data Fig. 2a). Also, injected lentiviruses did not travel to distant regions such as the lateral ventricle (Extended Data Fig. 2a). We ablated hypothalamic NSC through injection of lentiviruses expressing non-toxious herpes simplex virus thymidine kinase-1 (Hsv-TK) controlled by Sox 2 promoter and subsequently applying Ganciclovir (GCV) which was converted into a toxin by TK; hence, TK-expressing Sox 2 cells in hypothalamic $3 \mathrm{~V}$ wall were partially ablated. This treatment eventually led to $70 \%$ 
loss of Sox 2 cells in the $3 \mathrm{~V}$ wall of TK/GCV-injected mice without a major effect on parenchymal Sox 2 cells or neurons (Fig. 1c-e). For this model, we separately analyzed MBH neuronal subtypes POMC and AgRP neurons, using POMC-Cre and AgRP-Cre mouse stains. TK/GCV treatment did not decrease the numbers of these neurons (Extended Data Fig. 2b, c), and functionally optogenetic stimulation of each of these neuronal subtypes led to comparable feeding responses between TK/GCV-injected mice and controls (Extended Data Fig. 2d). To study aging-related physiology, TK/GCV model and various control groups were generated at a mid-aged condition. Compared to control groups over 3 4-month follow-up, TK/GCV mice displayed accelerated declines across muscle endurance, coordination, treadmill performance, sociality, and novel object recognition (Fig. 1f). Morris Water Maze test revealed that TK/GCV-injected mice manifested cognitive decline more than did controls (Fig. 1f, Extended Data Fig. 2e). In comparison with the regular speed of aging progression in intact mice (Extended Data Fig. 1), all these physiological changes in TK/GCV-injected mice reflected an acceleration in aging.

In parallel, we employed an alternative cell ablation method to test if it could reproduce the pro-aging phenotype in the TK/GCV model. According to the literature ${ }^{27}$, expression of inducible diphtheria toxin receptor (DTR) followed by DT administration has also often been used to ablate cells in animals. Thus, we generated lentiviruses that expressed DTR controlled by Sox 2 promoter, as indicated in Extended Data Fig. 3a and b. We then injected this DTR vs. control lentivirus into the hypothalamic $3 \mathrm{~V}$ of mid-aged mice, followed by DT administration which activated DTR. This approach resulted in a significant ablation of Sox2 cells in hypothalamic $3 \mathrm{~V}$ wall while MBH parenchymal cells were barely affected (Extended Data Fig. 3c, d). Indeed, DTR/DT treatment gradually led to accelerated physiological declines (Extended Data Fig. 3e), much as similarly as seen in the TK/GCV model described above.

Additional experiments were performed through intra-MBH viral injection, through which the ablation of hypothalamic NSC was restricted within the $\mathrm{MBH}$ portion of the $3 \mathrm{~V}$ wall and the MBH parenchyma. To complement with Sox2 promoter-based targeting in Fig. 1, we employed Bmil promoter to direct TK expression. We delivered TK vs. control lentiviruses bilaterally into the MBH of mid-aged mice (Fig. 2a), which led to a significant loss of NSC in the $\mathrm{MBH}$ portion of $3 \mathrm{~V}$ wall and the parenchyma (Fig. 2b, c), while the numbers of neurons in the MBH did not decrease (Fig. 2d). Clearly, compared to controls over 3 4month follow-up, TK/GCV-injected mice showed aging-like physiological declines (Fig. 2eh), cognitive impairment (Fig. 2i, j), and histopathology (Fig. 2k). Also, we set up a cohort of 8-month-old mice that were injected with TK vs. control lentiviruses for lifespan followup. This model was based on Sox 2 promoter-driven TK expression and MBH injection (Fig. 21, m). As shown in Fig. 2n, there was a significant decrease in the lifespan of TK/GCVtreated mice compared to controls. In summary, results from these several additional models consistently indicate that the MBH is crucial for the role of hypothalamic NSC in controlling the speed of aging. 


\section{Slowdown of aging by hypothalamic implantation of IxBa-htNSC}

With great interest, we simultaneously investigated if hypothalamic NSC could be implanted into the MBH to slow down aging progression. For the initial trial, we employed a line of newborn mice-derived hypothalamic NSC, labeled with GFP via lentiviral induction as described previously ${ }^{19}$, and injected them into the MBH of mid-aged mice. However, these NSC survived poorly following injection. This failure could be related to an inflammatory hypothalamic environment ${ }^{5}$, as we had appreciated that hypothalamic inflammation is hostile for hypothalamic $\mathrm{NSC}^{19}$. Hence, we asked if an inhibition of inflammatory response in these cells might help overcome this survival problem. To do so, we employed newborn mice-derived hypothalamic NSC that stably expressed dominant-negative I $x \mathrm{Ba}$ and GFP (IkBa-htNSC), and we had previously shown that these cells were resilient to NF- $\kappa \mathrm{B}-$ mediated inflammation, compared to control hypothalamic NSC that stably expressed GFP (Con-htNSC) ${ }^{19}$. We implanted the same number of I $x$ Ba-htNSC vs. Con-htNSC into the $\mathrm{MBH}$ of mid-aged mice. I $\mathrm{Ba}$-htNSC demonstrated a dramatic improvement in survival (Fig. 3a), showing that $\sim 50 \%$ grafted I $\mathrm{BBa}$-htNSC were alive while grafted Con-htNSC were barely found after 2 months post implantation. Then, we set up a group of mid-aged C57BL/6 mice injected in the MBH bilaterally with IkBa-htNSC to study aging-related physiology. For comparison, in addition to vehicle injection, a group of age-matched mice was injected with the same number of hypothalamic astrocytes. Hypothalamic astrocytes were derived from new-born mice, cultured and confirmed via immunostaining for the presence of astrocyte marker S100B and the absence of NSC markers. In addition, we included mesenchymal stem cells (MSC) as another type of comparison, as MSC are embryonically derived from the mesoderm layer and represent stem cells of a different origin. All these cells were genetically labeled with GFP, and the same number of these cells were bilaterally injected. Of note, without anti-inflammatory genetic manipulation, implanted astrocytes and MSC significantly survived to a comparable level as did I $\mathrm{kBa}$ htNSC (Fig. 3b). Compared to vehicle controls, implanted I $k$ Ba-htNSC led to a battery of systemic benefits which were already recognizable at 6 weeks post implantation and continued to be more appreciable at 4 months post implantation (Fig. 3c); in contrast, no evident effects were identified from the implantation of astrocytes or MSC (Fig. 3c). Independently, we generated separate groups of mid-aged mice injected in the MBH with I $\mathrm{Ba}$-htNSC for lifespan follow-up, while the control groups were injected with controlhtNSC or the vehicle. Control-htNSC did not offer a significant effect in lifespan compared to vehicle control (data not shown), agreeing with the fact that these cells did not survive long enough. In contrast, consistent with the anti-aging manifestations (Fig. 3c), mice injected with IrBa-htNSC demonstrated an increase in the lifespan (Fig. 3d). To summarize, a local restoration of hypothalamic NSC in mid-aged mice can provide a significant antiaging and longevity effect.

\section{Endocrine secretion of exosomal miRNAs by hypothalamic NSC}

As described above, the modulation of aging by hypothalamic NSC, when ablated or implanted, was observable in a relatively short period ( 3 to 4 months), thus we reasoned that an endocrine function rather than neurogenesis of these cells was accountable. Considering that the pool of hypothalamic NSC is small, yet the effects on modulating aging are 
substantial, we asked if these cells might secrete substances which could have multifaceted functions. We considered secretory miRNAs, as recent research has emerged to link miRNAs to stem cell function ${ }^{28,29}$ as well as aging ${ }^{30}$. Our prediction was supported by immunostaining with exosomal protein CD81 and electronic microscopic analysis, showing that granule-like structures (Extended Data Fig. 4a) and multivesicular bodies (Extended Data Fig. 4b) were abundantly present in hypothalamic NSC, but were poorly detected in the astrocytes from the same hypothalamus. Then, we isolated secretory exosomes from cultured hypothalamic NSC, as verified through a list of assays including Western blotting (Fig. 4a), flow cytometry (Extended Data Fig. 4c) and nano-particle analysis (Fig. 4b, Extended Data Fig. 4d). Secretory exosomes that we isolated were also subjected to fractioning by density gradient ultracentrifuge or pull-down via anti-CD81 antibody (Extended Data Fig. 5), both confirming that miRNAs were detected only in exosome fractions. Nano-particle analysis was further used to compare exosomes secreted from hypothalamic NSC vs. other cells, showing that (Fig. 4b) secretory exosomes from hypothalamic NSC were strikingly more abundant compared to secretion by astrocytes, GT1-7 and MSC. Using Qubit RNA assay to quantitate exosomal RNAs, we found that secretory exosomes of hypothalamic NSC contained a large amount of RNA. We analyzed miRNA in these secreted exosomes, showing that hypothalamic NSC produced miRNA more abundantly than did hippocampal NSC, and 100-fold times more than did hypothalamic astrocytes, GT1-7 cells and MSC (Fig. 4c, Extended Data Fig. 6a, b). To provide more details, we profiled secretory exosomal miRNAs of these different cells using microarray affymetrix chips, showing that hypothalamic NSCs were uniquely potent in secreting exosomal miRNAs (Fig. 4d). Despite that hippocampal NSC also produced a large amount of exosomes, exosomal miRNAs from these cells were lower and displayed different patterns compared to hypothalamic NSC (Fig. 4e). Thus, hypothalamic NSC have an important endocrine function of secreting exosomal miRNAs.

\section{Contribution of hypothalamic NSC to brain's exosomal miRNAs}

Using information from miRNA microarray affymetrix chips (Fig. 4d), we analyzed miRNAs in the CSF samples collected from young vs. mid-aged mice. Focusing on miRNA species which are abundantly produced by hypothalamic NSC, we found that over 20 miRNA species substantially decreased in the CSF of mid-aged mice (Fig. 5a). To test if the reduction of these miRNAs in the CSF of mid-aged mice could be due to aging-associated loss of hypothalamic NSC, we designed an experiment in which exosomal secretion of hypothalamic NSC was genetically inhibited. To do so, we created a lentivirus containing Cre-dependent shRNA against Rab27a, a key molecule for the function of exosomal secretion. Through co-infection with Sox 2 promoter-driven Cre lentivirus, and using two independent shRNA sequences against Rab27a mRNA, we confirmed that this approach led to reduced secretion of exosomal miRNAs in cultured hypothalamic NSC (Fig. 5b). For comparison, we analyzed a list of growth factors and cytokines but found that Rab27a shRNA did not affect secretion of a majority of these peptide hormones from htNSC (Extended Data Fig. 7). This inhibitory effect on exosomal release by Rab27a shRNA was not a result of cell loss, as Rab27a shRNA did not immediately affect the survival or proliferation of these cultured cells (Extended Data Fig. 8a, b). Therefore, we injected Sox2- 
Cre lentivirus together with Rab27a shRNA vs. control shRNA lentivirus into the hypothalamic 3V of young mice. One week later, CSF samples were examined, showing that Rab27a shRNA resulted in decreases in many miRNAs in the CSF (Fig. 5c). We applied this approach to mid-aged mice and analyzed aging-related physiology at 6 weeks post injection, during which Rab27a shRNA did not yet cause a reduction in hypothalamic NSC (but it did so over a long duration such as 3 months). As shown in Extended Data Fig. 8c, mice injected with Rab27a shRNA showed impairments in several aspects of physiology, although to lesser degrees compared to the models of cell ablation.

\section{Slowdown of aging by hypothalamic NSC-derived exosomes}

The results above supported a role of exosomal secretion from hypothalamic NSC in the control of aging. Although we also predict that neuropeptide secretion by these cells is relevant, especially in the context of our recent finding that GnRH is involved in hypothalamic control of aging ${ }^{5}$, we have clearly observed that implanted hypothalamic NSC led to expression of GnRH (data not shown); however, we decided to focus this work on hypothalamic NSC-secreted exosomes. To do so, we designed to test if aging could be slowed down by treatment with purified exosomes as verified via electron microcopy (Extended Data Fig. 9). First, following observation that exosomal miRNAs are transferrable among hypothalamic NSC (Fig. 6a), we confirmed that central treatment with these exoxomes in mid-aged mice helped maintain hypothalamic NSC (Fig. 6b), and this effect was associated with a reduction in hypothalamic inflammation (Extended Data Fig. 10a). Then, physiological experiments were performed, using an NSC ablation-induced aging model and a normal aging model. To do so, mid-aged mice with TK/GCV-induced ablation of hypothalamic NSC as described in Fig. 2 received 3-month treatment with secretory exosomes purified from cultured, newborn mice-derived hypothalamic NSC. While TK/ GCV-induced ablation of NSC in control group led to pro-aging effects, many of these changes were ameliorated by the treatment with exosomes (Fig. 6c, Extended Data Fig. 10b). These anti-aging effects were not a result of food intake change, as these mice ate similarly as did controls. In parallel, we performed an experiment in which mid-aged C57BL/6 mice were treated with exosomes vs. vehicle for 4 months. Compared to the vehicle-treated group which showed many aging-associated disorders, exosome-treated mice developed these problems to much lesser degrees (Fig. 6d, Extended Data Fig. 10c), and these protective effects were also independent of food intake change. Based on these therapeutic experiments, exosomal miRNAs are important for the role of hypothalamic NSC in the control of aging.

\section{Discussion}

The results in this work indicate that loss of hypothalamic NSC is an important cause for the whole body's aging. This understanding aligns with our recent discovery that hypothalamus has a programmatic role in systemic aging 5 . Logically, the underlying basis could be related to two functions of these cells: endocrine secretion and neurogenesis. Here we reported that the modulation of aging by hypothalamic NSC was achieved in a relatively short period which should not have a major contribution from neurogenesis while an endocrine function of these cells provided as a neurogenesis-independent mechanism. In this context, we 
revealed that the anti-aging effect of hypothalamic NSC is partially mediated by exosomal miRNAs secreted from these cells. Thus, besides the classical function of the hypothalamus in secreting neuropeptides, hypothalamic NSC bear a new type of endocrine function through secreting exosomal miRNAs. Given this finding, we also hope to express that neuropeptide secretion by hypothalamic NSC, although not studied in this work, is also predicted to participate in regulation of systemic aging, partly because we found that implanted hypothalamic NSC gave rise to GnRH-expressing cells -which aligns with our previous finding that $\mathrm{GnRH}$ is involved in hypothalamic control of aging 5 . Also, we predict that hypothalamic NSC-mediated neurogenesis contributes to the long-term, anti-aging effects of these cells, which warrants future studies. Despite these outstanding questions, the overall findings in this work conclusively support the point that hypothalamic NSC is crucial for the control of aging speed.

\section{Methods}

\section{Cell culture}

Primary culture of NSC were performed as described previously ${ }^{19}$. Briefly, the hypothalamus and hippocampus were dissected from new-born C57BL/6 mice, cut into small pieces $\left(\sim 1 \mathrm{~mm}^{3}\right)$, and followed by digestion via TrypLE Express enzyme (Life Technologies) for $30 \mathrm{~min}$ at $37^{\circ} \mathrm{C}$. After centrifugation, cells were suspended in NSC medium composed of Neurobasal-A (Life Technologies), 0.24\% GlutaMAX ${ }^{\mathrm{TM}}$ Supplement (Life Technologies), 2\% B27 without vitamin A (Life Technologies), 10 ng/ml EGF (Sigma), $10 \mathrm{ng} / \mathrm{ml}$ bFGF (Life Technologies) and 1\% penicillin-streptomycin and seeded in ultralow adhesion 6-well plates (Corning), and one week later, neurospheres were collected via centrifugation and trypsinized through TrypLE Express enzyme into single cells, passed and maintained in neurosphere culture until experimental use. Hypothalamic astrocytes were isolated from neonatal C57BL/6 mice as described previously ${ }^{31}$, briefly, hypothalamus of neonatal mice was dissected, the meninges were removed and cells were dissociated in $0.05 \%$ trypsin-EDTA. Mixed cells were cultured at $37^{\circ} \mathrm{C}$ in $5 \% \mathrm{CO}_{2}$. Non-adherent cells were removed by changing the media every 3 days. After $7-10$ days, cells were shaken rigorously in an orbital incubator at $0.23 \mathrm{~g}$ at $37^{\circ} \mathrm{C}$ for 2 hours to detach microglia, then shaken at $0.23 \mathrm{~g}$ at $37^{\circ} \mathrm{C}$ overnight to remove oligodendrocytes. Remaining cells, which were most astrocytes, were trypsinized and reseeded for further culture. HEK293T cells were purchased from ATCC (Cat\# CRL-3216), and GT1-7 cells were established as previously reported ${ }^{32}$. HEK293T cells, GT1-7 cells and astrocytes were cultured in Dulbecco modified Eagle medium (DMEM) containing 10\% fetal bovine serum and 1\% penicillin-streptomycin. Mesenchymal stem cells were isolated from 6-8 week-old C57BL/6 mice as described previously ${ }^{33}$. Briefly, bone marrow cells were flushed out from femurs and tibias, and cultured in DMEM containing $15 \%$ fetal bovine serum and $1 \%$ penicillinstreptomycin at $37^{\circ} \mathrm{C}$ in $5 \% \mathrm{CO} 2.3$ hours later, medium were changed to remove nonadherent cells. After 1-2 weeks of culture, cells reached confluence and passed for subsequent experiments. All cell lines used in this study were free of microbial (including mycoplasma) contamination and their morphology and growth characteristics were compared to published information to ensure their authenticity. 


\section{Plasmids and recombinant lentiviruses}

Lentiviral vector of Sox 2 promoter-driven Hsv-TK and matched control were generated as reported previously ${ }^{34}$. Lentiviral vector of Bmil promoter-driven TK was generated by replacing Sox 2 promoter with Bmi1 promoter in the above TK plasmid. Lentiviral vector of Sox 2 promoter-driven DTR was generated by replacing TK cDNA with the coding sequence of simian DTR in the plasmid of Sox2 promoter-driven TK. Mouse Rab27a shRNA and scramble shRNA were constructed using the vector pSico (Addgene). Rab27a shRNA-1: GGAGAGGTTTCGTA-GCTTA, Rab27a shRNA-2: GCTTCTGTTCGACCTGACA, scramble shRNA sequence: ATCTCGCTTGGGCGAGAGT. Plasmids of rAAV2-FLEX-revChR2:tdTomato were purchased from Addgene. Lentiviruses were produced by transfecting viral plasmids and packaging plasmids into HEK293T cells, purified via ultra-centrifugation and titrated using p24 ELISA kit as described previously ${ }^{32}$.

\section{Lentiviral infection and miRNA transfection}

Lentiviral infection to cells were performed by adding purified lentiviruses into culture medium for 24 hours before the medium was replaced. Synthetic miRNAs were purchased from Qiagen, including miR-106a-5p, miR-20a-5p and miR-466m-5p, and labeled with Cy3 using Silencer siRNA labeling kit (Thermo Fisher Scientific, AM1632). $5 \mu \mathrm{g}$ miRNAs were incubated for 1 hour in the dark followed by precipitation of miRNAs through adding ethanol and $\mathrm{NaCl}$. Cy3-labeled miRNAs were transfected into cells via HiPerFect Transfection Reagent (Qiagen).

\section{Cultured cell immunostaining}

Cells were fixed with 4\% PFA, blocked with the serum of appropriate species, penetrated with $0.2 \%$ Triton $\mathrm{X}-100$, and subjected to reaction with primary antibodies followed by reaction with Alexa Fluor 488-, 555- or 633-conjugated secondary antibodies (Invitrogen). Images were captured by a confocal microscope, and counting analysis included at least three coverslips per group and multiple areas per slide on a random basis. Primary antibodies included rabbit anti-Sox2 (Millipore, cat\#AB5603), rabbit anti-CD81 (Santa Cruz, cat\#sc-9158), and mouse anti-DTR (Abcam, cat\#ab92620).

\section{Electron microscopy}

Electron microscopic experiment was performed at the St. Giles Foundation Advanced Microscopy Center, Cold Spring Harbor Laboratory (Cold Spring Harbor, NY). Cells were cultured in laminin-coated $35 \mathrm{~mm}$ polyestirene dish and fixed in $2 \%$ glutaraldehyde in 0.1 $\mathrm{mol} / \mathrm{L}$ PBS. Cells were post-fixed in situ with $1 \%$ osmium tetroxide and $1.5 \%$ potassium ferrocyanide in distilled water for 1 hour. Cells were then dehydrated in a grade series of ethanol and infiltrated with 50\% epon-araldite resin (Sigma Aldrich) for one hour and then infiltrated for 2 hours in $100 \%$ resin. Petri dishes were then filled with fresh resin and placed in a $600{ }^{\circ} \mathrm{C}$ oven overnight. The petri dish polystyrene was sawn off from the edges of the dish and the disk was plunged repeatedly in liquid nitrogen using differences in thermal expansion to peel off the polystyrene on the bottom of the dish from the epoxy block. The embedded cells were then mounted attachments surface side up in an ultramicrotome chuck and thin sectioned at $100 \mathrm{~nm}$. Thin sections were counterstained with lead citrate and 
examined in a Hitachi H7500 transmission electron microscope. Representative areas were recorded on Kodak 4489 negative film (Electron Microscopy Sciences) that was then scanned at 2400 DPI using an Epson V750 Pro scanner.

\section{Exosome isolation and analyses}

Secretory exosomes in culture media were purified via differential centrifugations as described previously ${ }^{35}$. Briefly, culture medium was processed via ultracentrifuge at $4^{\circ} \mathrm{C}$ overnight to remove particles to generate exosome-free medium. Cells were cultured in an exosome-free medium for 2 days, media were collected, centrifuged to remove cells, immediately followed by exosome isolation in $4^{\circ} \mathrm{C}$. Exosome-containing media were centrifuged and filtered to remove debris and particles. Exosomes in filtered media were isolated by differential centrifugations. Un-cultured media of the same volume were processed with the same purification procedures and used as a technical control. Isolated exosomes were assessed according to a list of different approaches, including: 1) Exosomal protein marker immunoblot: Exosomal proteins vs. cellular proteins were loaded and separated in SDS-PAGE, followed by Western blotting with primary antibodies including mouse anti-TSG101 (Santa Cruz, cat\#sc-7964), rabbit anti-CD81 (Santa Cruz, cat\#sc-9158), rabbit anti-AGO2 (Cell Signaling, cat\#2897), rabbit anti-HSP90B1 (Cell signaling, cat\#2104), mouse anti-GM130 (Santa Cruz, cat\#sc-55591), and rabbit anti-Cyc1 (Cell Signaling, cat\#4272). Silver staining was performed using ProteoSilver Silver Stain Kit (Sigma). 2) Flow cytometry: Purified exosomes were incubated with latex beads (Life technologies) for $15 \mathrm{~min}$, adjusted to a final volume, and rotated at room temperature for 2 hours, and added with glycine. After incubation, beads were collected by centrifugation, resuspended and FACS staining using FITC-conjugated anti-CD81 (ThermoFisher, MA5-17939) antibody. 3) Nanoparticle tracking: Purified exosomes were run on NanoSight (Malvern Instruments), performed in Center for Nanotechnology in Drug Delivery at University of North Carolina. 4) Density-gradient ultracentrifugation analysis: Isolated exosomes were re-suspended in 2.5 M sucrose solution (mM HEPES, pH7.4), plated with continuous sucrose gradient from $2 \mathrm{M}$ to $0.25 \mathrm{M}$, and ultracentrifuged overnight at $4^{\circ} \mathrm{C}$. Different fractions were collected, each fraction recovered by ultracentrifugation and analyzed by immunoblot and real-time PCR. 5) Pull-down assay: Anti-CD81 antibody and isotype control antibody were coated onto Protein G Beads (Life Technologies, 10003D), exosomes were re-suspended in PBS containing $3 \mathrm{mg} / \mathrm{ml} \mathrm{BSA}$ and incubated with antibodycoated beads overnight with rotation at $4{ }^{\circ} \mathrm{C}$, and subsequently beads were washed with the same buffer. Isolated exosomes were analyzed by real-time PCR. 6) Exosomal total protein and RNA: Exosomal total protein concentration was measured by Pierce ${ }^{\mathrm{TM}}$ Coomassie (Bradford) Protein Assay Kit (ThermoFisher). Exosomal total RNAs and small RNAs were purified by mirVana miRNA Isolation Kit (Life Technologies). Exosomal total RNAs were measured by Qubit assay (ThermoFisher).

\section{Exosomal miRNA analyses}

Exosomal miRNAs were analyzed according to the following approaches: 1) Exosomal small RNA bioanalyzer analysis: Exosomal RNAs were run on pico RNA chips or small RNA chips of Agilent 2100 Bioanalyzer system (agilent technologies), performed in the Molecular Pathology Platform, Herbert Irving Comprehensive Cancer Center, Columbia 
University, New York. Exosomal miRNA concentration was calculated according to the instruction provided by agilent technologies. Briefly, the percentage of miRNAs in exosomal total RNAs was calculated by bioanalyzer pico RNA chips and small RNA chips, and miRNAs concentration was calculated by multiplying $\%$ of miRNAs by exosomal total RNAs measured by Qubit assay (ThermoFisher). 2) miRNA microarray: miRNAs microarray was performed on GeneChip® miRNA 4.0 Array (Affymetrix) in the Albert Einstein College of Medicine Genomics Core using exosomal small RNAs as input. miRNAs pathway analysis was performed using DIANA-mirPath v.3 online software. 3) miRNA quantitative PCR: miRNAs were extracted from purified exosomes or the CSF using mirVana $^{\mathrm{TM}}$ miRNA isolation kit (Invitrogen) or miRNeasy Mini Kit (Qiagen). Extracted miRNAs were reverse transcripted to cDNA using miRNA first-strand cDNA synthesis kit (Invitrogen or Agilent Technologies), and then subjected to real-time PCR with specific primers and SYBR ${ }^{B}$ Green PCR Master Mix (Thermo Fisher Scientific).

\section{Secretory growth factor chip array}

Cell models of NSC were cultured in media without containing EGF and bFGF for 2 days, and media were collected and analyzed using Mouse Growth Factor Array (RayBiotech, AAM-GF-2) according to the manufacturer's protocol. Array images were collected and analyzed by LI-COR Odyssey Fc imaging system.

\section{Animal models}

C57BL/6 mice were obtained from the Jackson Laboratory and the National Institute of Aging. AgRP-Cre mice and POMC-Cre mice were maintained on C57BL/6 strain background as we had previously reported ${ }^{32,36}$. All mice were kept in standard, infectionfree housing conditions, with 12 light/12 dark cycles and 4-5 mice per cage. Strictly pathogen-free quality of mouse colonies was maintained through quarterly serology, quarterly histopathologic exams and daily veterinarian monitoring on general health and care of animals. All mice in this study were kept on a standard normal chow diet obtained from LabDiet (Cat\# 5001, 4.07 Kcal/gram). Only male mice were reported in this study. All procedures were approved by the Institutional Animal Care and Use Committee of the Albert Einstein College of Medicine.

\section{Hypothalamic lentiviral injection}

Purified lentiviruses suspended in 0.2- $\mu \mathrm{l}$ artificial CSF (aCSF, Tocris Bioscience) were injected bilaterally into the MBH under an ultra-precise stereotactic apparatus (David Kopf Instruments) using the coordinate of $1.7 \mathrm{~mm}$ posterior to bregma, $5.8 \mathrm{~mm}$ below the surface of skull, and $0.25 \mathrm{~mm}$ lateral to the midline of the brain, via a 26-gauge guide cannula and a 33-gauge internal injector (Plastics One) connected to a 5- $\mu$ l Hamilton syringe and infusion pump (WPI Instruments). Lentiviruses suspended in 0.4- $\mu$ aCSF were injected into the hypothalamic third ventricle using the coordinate of $1.7 \mathrm{~mm}$ posterior to bregma, $5 \mathrm{~mm}$ below the surface of skull. Guide cannula was implanted into the third ventricle of mice at the coordinate of $1.7 \mathrm{~mm}$ posterior to the bregma and $5.0 \mathrm{~mm}$ below the surface of skull. 


\section{Chemical treatment and cell implantation}

Mice with MBH injection of TK-expressing lentivirus were treated with GCV (1-10 $\mu \mathrm{g}$ in $0.5 \mu \mathrm{laCSF}$ ) vs. vehicle via pre-implanted third-vehicle cannula, twice per week for the first month (or the experimental duration if less than 1 month) and then weekly afterwards throughout the experiment. Mice with MBH injection of DTR-expressing lentivirus were i.p. injected with DT ( $25 \mu \mathrm{g} / \mathrm{kg}$ body weight in saline) vs. the vehicle, twice per week throughout the experiment. For BrdU tracking experiment, mice were injected with BrdU (1 $\mu \mathrm{g}$ in $0.5 \mu \mathrm{laCSF}$ ) via pre-implanted cannula twice per day for one week. Exosome treatment was performed by using exosomes (100 ng protein, purified from cultured hypothalamic NSC of newborn mice) 3 times per week for a duration of 3-4 months as indicated. Cell implantation was performed as described previously ${ }^{19}$. Briefly, cultured cells suspended in $0.5 \mu \mathrm{l}$ phosphate buffer saline were bilaterally injected into the MBH $(9,000$ cells each MBH side) using the coordinates of $1.7 \mathrm{~mm}$ posterior to bregma, $5.8 \mathrm{~mm}$ below the surface of skull, and $0.25 \mathrm{~mm}$ lateral to the midline of the brain via a 26-gauge guide cannula and a 33-gauge internal injector connected to a 5- $\mu 1$ Hamilton syringe and infusion pump.

\section{Behavioral tests}

All behavior tests are performed in behavioral testing room. An Anymaze video tracking system (Stoelting) equipped with a digital camera connected a computer was used to the whole course of animal activities in training and experimental sessions of behavioral tests, including: 1) Grip test (muscle endurance): As described previously ${ }^{37}$, each mouse was lifted by the tail and placed on a homemade square grid (1-cm mesh size), the grid was then inverted $30 \mathrm{~cm}$ over a soft pad, and the mouse was allowed to hang by paws for 2-6 min according to age conditions. The time that the mouse was able to hang was recorded during a 2-min test period. Three times of repeats were performed for each mouse with at least 10 minutes rest between each trial. 2) Rotarod test (coordination): Mouse was trained on a rota rod (Columbus instruments) moving at a constant speed of 6 rpm for 60 seconds. After 10min rest, each mouse was given three trials, during which the rota rod started at $6 \mathrm{rpm}$ and accelerated to $2 \mathrm{rpm}$ per minute until $10 \mathrm{~min}$, and there was 30-min rest period between each trial. 3) Treadmill: Treadmill test was performed using Treadmill Simplex II (Columbus instruments), capability of exercising up to six mice simultaneously in individual lanes. Mice were warmed up for protecting running injury and failure before experimental running. For acclimation of treadmill, subject mouse was placed at a slow speed $(6 \mathrm{rpm} / \mathrm{min})$ of treadmill running for $5 \mathrm{~min}$. The test session started at $8 \mathrm{rpm}$ for subject mouse running, and increased $2 \mathrm{rpm}$ every min for $10 \mathrm{~min}$. The time which the subject mouse retired was recorded. 4) Sociality: Using a standard procedure as described ${ }^{38}$, social interaction was tested in a gray 3-chamber neutral box cage (plexiglas, $60-\mathrm{cm}$ length, $40-\mathrm{cm}$ width, $22-\mathrm{cm}$ height). Before first session, each mouse was allowed to explore freely for $5 \mathrm{~min}$ in the neutral cage to habituate to the testing conditions (adaptation). During the first session (social affiliation), a new mouse (Stranger 1) was placed in a wire containment cup that was located in one side chamber. Subject mouse was allowed free access to explore each of the three chambers for $10 \mathrm{~min}$. During the second session (preference), a second new mouse (Stranger 2) placed to a wire containment cup was put in the opposite side chamber. The subject mouse freely explored each of the three chambers for $10 \mathrm{~min}$. The time spent in 
social interaction (sniffing) was recorded. 5) Novel object recognition: Object recognition test was conducted as described in literature ${ }^{39}$. An individual mouse freely explores to an open-field box ( $40 \mathrm{~cm}$ length $\times 40$ width and $\times 50 \mathrm{~cm}$ height) for $10 \mathrm{~min}$ before experimental sessions. During first session (familiarization session), the mouse was allowed to freely explore two similar objects, and during the second session (test session), one of two objects was replaced by a novel object for $10 \mathrm{~min}$. The time which mouse spent to explore each object was recorded. A preference index was calculated using the ratio of the amount of time spent exploring any one of the two objects (including novel one) over the total time spent exploring both objects. 6) Morris water maze (MWM): Morris water maze test was performed as described ${ }^{5}$. The water tank was filled with $22-23^{\circ} \mathrm{C}$ water, crayola non-toxic paint was added to make opaque and white background, and was located in the center of a small room with extra-maze cues (various black shapes on white background). The diameter of the maze was $90 \mathrm{~cm}$ and divided into four quadrants (northwest, northeast, southwest and southeast). A circular platform with a diameter of $10 \mathrm{~cm}$ was placed $25 \mathrm{~cm}$ from the wall of the tank. A mouse was placed on water, at the same starting location for all trials, and was measured for latency, distance and speed travelled to the platform. Hidden-platform training: mice were first required to swim to and sit on a circular visible platform at $0.5 \mathrm{~cm}$ above water level for 10 seconds. If mouse could not find the platform within 60 seconds, it was gently guided to the platform using a glass stirring rod. Mouse was then subjected to 4 consecutive days of training, consisting of a trial per entry location (entry locations were north, south, east and west) for a total of four trials per day. The platform was made invisible by submerging it $1 \mathrm{~cm}$ below the surface of the water. Mice were expected to find the location of the invisible platform, and measured for latency to reach the platform, distance travelled to reach the platform, path efficiency, time spent in and distance travelled in each quadrant as well as total distance and swimming speed. Probe trial: on day 5, mouse was subjected to a single probe trial, in which the platform was removed and animal was allowed to swim for 60 seconds. Mouse was measured for the amount of time spent in all quadrants, distance and times that mouse crossed the location of the former platform, total distance and swimming speed. The learning and memory of mice were indicated positively by occupancy time (the total time spent in target quadrant) in probe trials.

\section{Tissue immunostaining, CSF collection, and histology}

Mice under anesthesia were transcardially perfused with 4\% PFA, and then brains were removed, post-fixed, and infiltrated with $20-30 \%$ sucrose. For immunostaining, brain sections in 20- $\mu \mathrm{m}$ thickness were cut using cryostat, blocked with serum of appropriate species, treated with primary antibodies, including mouse anti-Sox2 (R\&D Systems, cat\#MAB2018), rabbit anti-Sox2 (Millipore, cat\#AB5603), mouse anti-Bmi1 (Abcam, cat\#ab14389), mouse anti-Nestin (Millipore, cat\#MAB353), mouse anti-BrdU (Cell Signaling, cat\#5292), rabbit anti-Ki67 (Abcam, cat\#ab15580), rabbit anti-Hsv-TK (Santa Cruz, cat\#sc-28038), mouse anti-GFP (Thermo Fisher Scientific, cat\#MA1-952), and subsequently reacted with Alexa Fluor 488 or 555 secondary antibodies. Technical controls for antibody reaction included using naïve IgGs of appropriate species. DAPI staining for sections was used to reveal all comprised cells. For BrdU staining, tissue sections were pretreated with $2 \mathrm{~N} \mathrm{HCl}$ for $20 \mathrm{~min}$ at $30^{\circ} \mathrm{C}$, washed and followed by incubation with $0.1 \mathrm{M}$ borate buffer for $10 \mathrm{~min}$ at room temperature. Fluorescence images of sections were 
captured under a con-focal microscope. Cells of interest in tissue immunostaining were counted in one of every 3-6 serial sections for the whole target region or a representative sub-area, and then the total number of cells was calculated by multiplication according to these factors (which was predicted to be proportional to the actual total number of cells in a 3-dimenstional hypothalamic region). CSF was collected from anesthetized mice by penetrating a pulled capillary tube into the cisterna magna through dura mater, as previously described $^{40}$, and the fluid from 3 to 4 mice were pooled as an individual sample, and the same volume of all samples were used for miRNA analysis. With skills, extra caution was taken to avoid any contamination from the blood or tissue during the procedure of collecting the CSF. For tissue histology, skeletal muscles (quadriceps) and dorsal skin dissected from mice were taken and fixed in $10 \%$ neutralized formalin at $4^{\circ} \mathrm{C}$ overnight and embedded into paraffin. Paraffin sections were then prepared at 5- $\mu \mathrm{m}$ thickness and subjected to haematoxylin and eosin staining.

\section{Optogenetics}

According to the established protocol in the literature ${ }^{41}$, AgRP-Cre and POMC-Cre mice received a hypothalamic third-ventricle injection of Hsv-TK lentiviruses and an intra-MBH bilateral injection of $0.2-\mu \mathrm{l}$ rAAV2-FLEX-rev-ChR2:tdTomato viruses $\left(1 \times 10^{13}\right.$ genomic copies per ml, produced from Applied Biological Materials Inc.). Optic/Fluid Cannula (Doric Lenses) was implanted into the hypothalamic third ventricle of mice at the coordinate of $1.7 \mathrm{~mm}$ posterior to the bregma and $5 \mathrm{~mm}$ below the surface of skull. Mice received GCV treatment through the implanted cannula. At experiment, light from a diode laser $(450 \mathrm{~nm}$, Doris Lenses) was delivered to the brain via an optic fiber ( 0.22 NA, $200 \mu \mathrm{m}$ core; Doric Lenses), as previously described ${ }^{41}$, including 20 light pulses (10 ms each) for 1 second (20 $\mathrm{Hz}$ ) followed by a 3-second break, and the sequence repeated for 1 hour in AgRP-Cre mice and 24 hours in POMC-Cre mice. Optogenetic stimulation-induced feeding response was assessed through measuring food intake during various time intervals.

\section{Statistics and Reproducibility}

Kolmogorov-Smirnov test was used to analyze normal distribution of data, two-tailed unpaired Student's t-test was used for analyses which involved only two groups for comparison, and ANOVA and appropriate post-hoc test were used for analyses which involved more than two groups for comparisons. Lifespan curves were analyzed with Kaplan-Meier survival curve and $p$ values were obtained using log-rank (Mantel-Cox) test. Data presented met normal distribution, data variance among comparable experimental groups was similar, and statistical tests for each figure were justified appropriate. Sample sizes were chosen with adequate power based on the literature as well as our previous studies, in general without using statistical methods to predetermine sample size. Animals were randomized into different groups with approximately comparable numbers of animals each group whenever possible. No samples or data points were arbitrarily excluded from statistical analysis. Experimental performers were generally not blind to group information. Key experiments including mouse models of hypothalamic NSC ablation and hypothalamic NSC implantation and their behavioral analyses, exosomal miRNA experiments including microarray, small RNA/miRNA bio-analysis, nano-particle analysis and qPCR, and mouse 
CSF sampling and analysis, were all independently repeated with similar observations. All data were presented as mean \pm s.e.m., and $p<0.05$ was considered statistically significant.

\section{Data availability}

All source data supporting the findings of this study are available within the paper and its supplemental files. Microarray data are also made available in a supplemental file. 


\section{Extended Data}
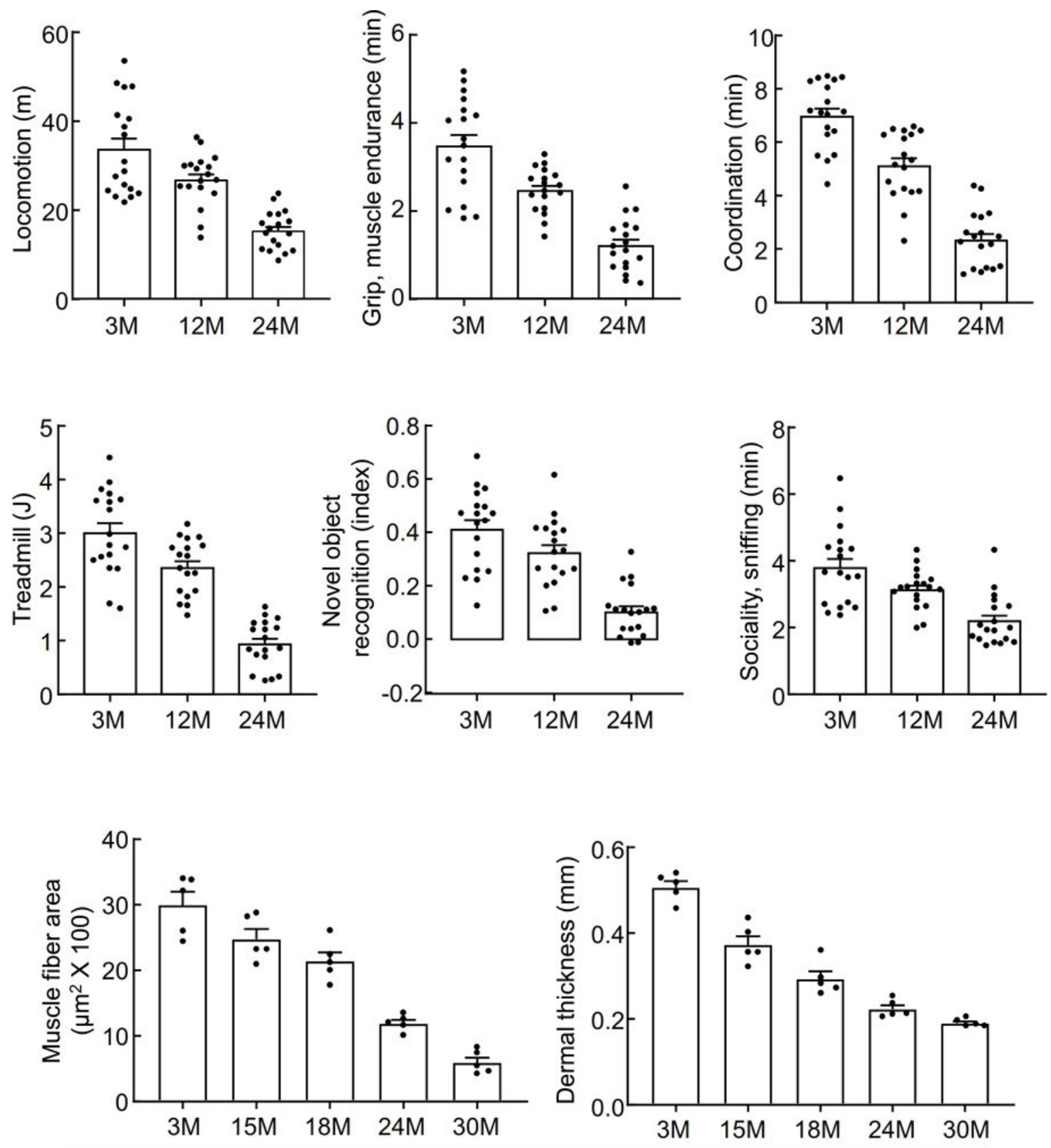

Standard C57BL/6 males, age (Months, M)

Extended Data Figure 1. Aging-related physiology and histology in C57BL/6 mice

Male C57BL/6 mice at indicated ages (months, M) were maintained under standard housing and chow feeding conditions without any experimental treatment except being analyzed for aging-related physiological parameters and tissue histology as indicated. $\mathrm{n}=18$ mice per group (behaviors) and $\mathrm{n}=5$ mice per group (histology). Error bars reflect mean \pm s.e.m. 

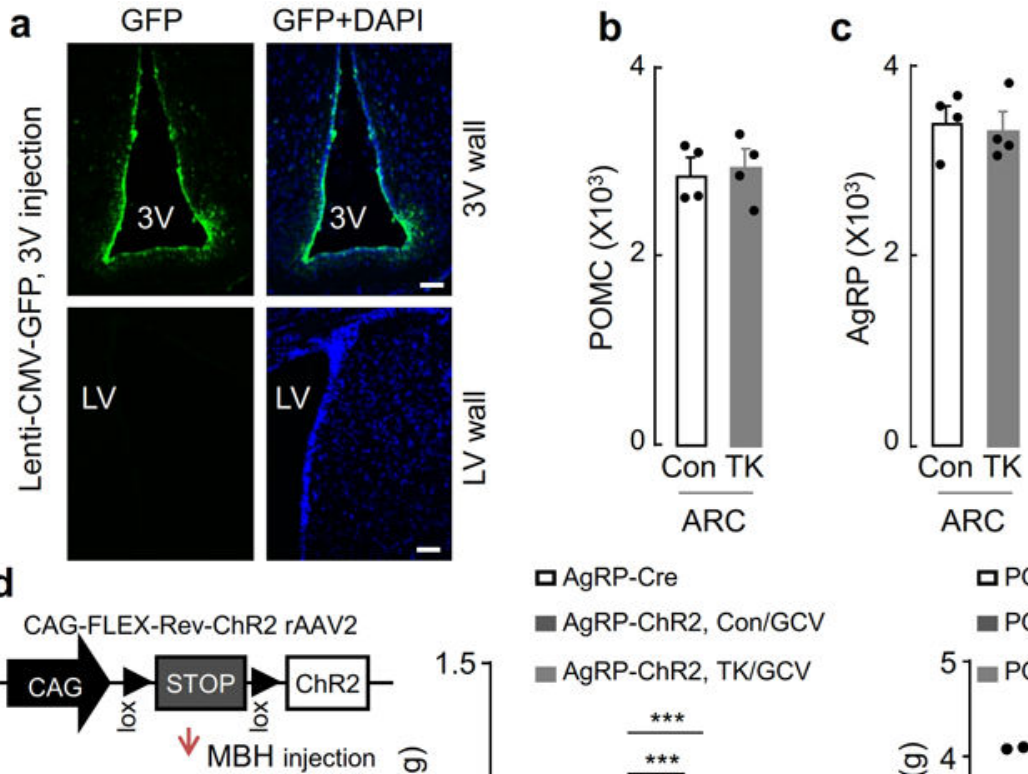

- AgRP-ChR2, Con/GCV
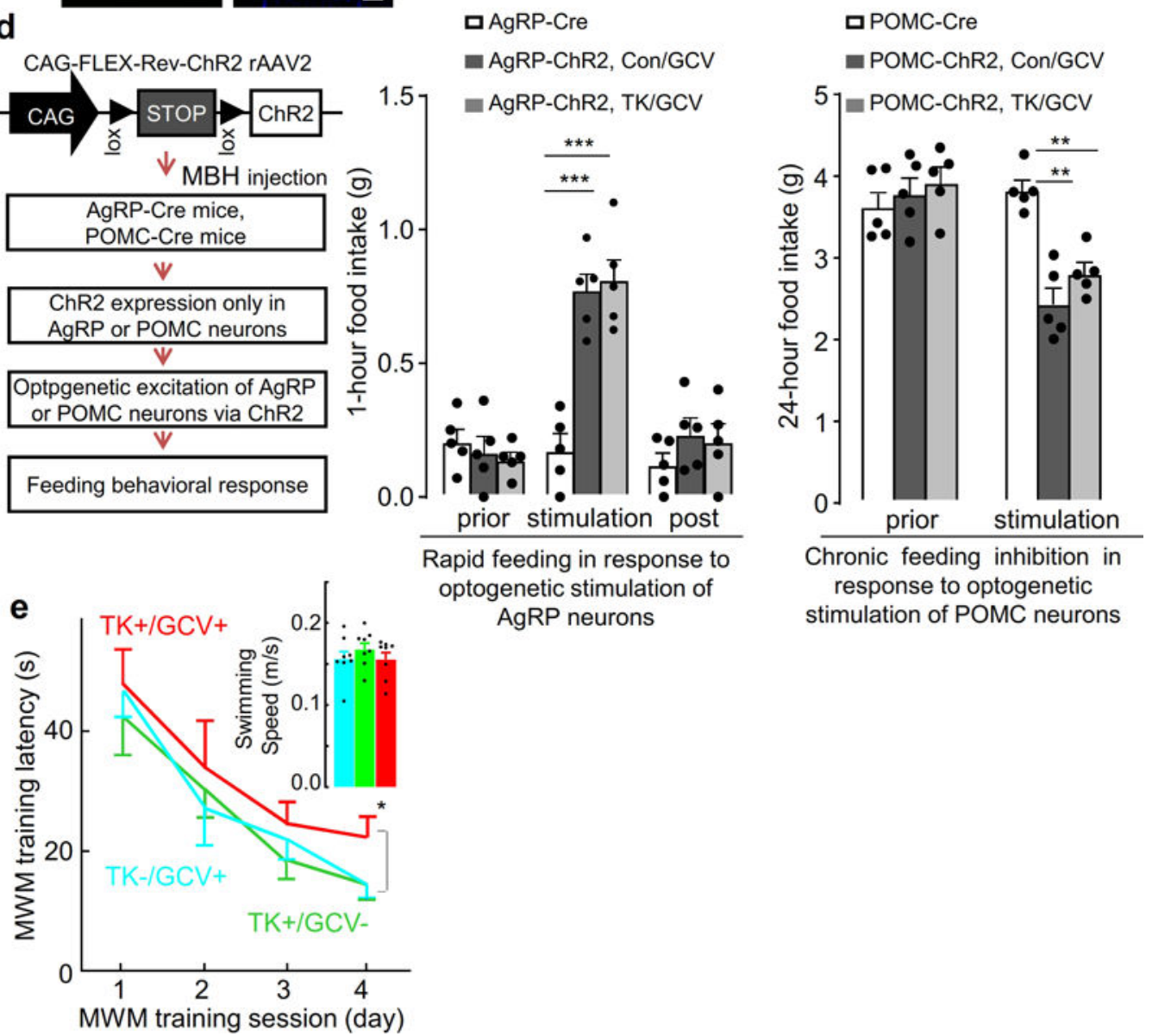

a AgRP-ChR2, TKJGCV

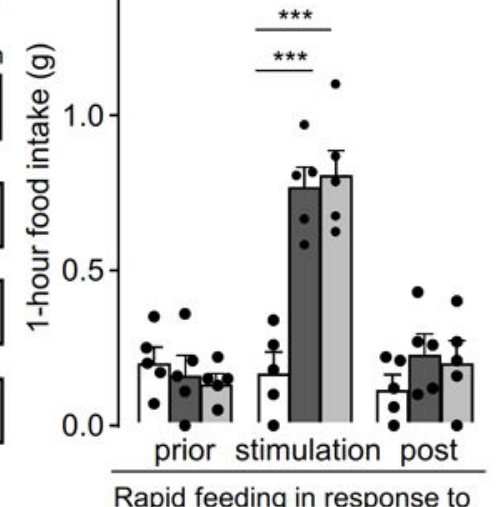

optogenetic stimulation of AgRP neurons stimulation of POMC neurons

Extended Data Figure 2. Viral injection and additional information on TK/GCV model a, Lentiviruses of CMV-promoter driven GFP were injected into the hypothalamic third ventricle (3V) of C57BL/6 mice via pre-implanted cannula. At 1 week after injection, brain sections were made and examined for GFP immunostaining. Scale bar, $50 \mu \mathrm{m}$. b, c, AgRPCre mice and POMC-Cre mice received hypothalamic 3V injection of Hsv-TK lentiviruses followed by GCV treatment and were examined 3 months later for the numbers of these neurons in the ARC through Cre immunostaining. d, AgRP-Cre mice and POMC-Cre mice were injected with rAAV2-FLEX-rev-ChR2:tdTomato viruses or vehicle into the ARC, 
followed by injection of Sox 2 promoter-driven Hsv-TK lentivirus (TK) or control lentivirus (Con) into the hypothalamic third ventricle. GCV was administrated into the third ventricle twice per week for 3 weeks. Subsequently these mice were subjected to optogenetic stimulation-induced feeding response as described in Method. Food intake prior to and post optogenetic stimulation were also measured. e, MWM training information for Figure 1f. Images represent 4 independent experiments $(\mathbf{a}) . * * \mathrm{p}<0.01,{ }^{* * *} \mathrm{p}<0.001$; two-tailed Student's t-test $(\mathbf{b}, \mathbf{c})$, one-way ANOVA with Tukey's post-hoc test $(\mathbf{d}, \mathbf{e}) ; \mathrm{n}=4$ mice per group $(\mathbf{b}, \mathbf{c}), \mathrm{n}=5$ mice per group $(\mathbf{d})$, and $\mathrm{n}=8$ mice per group $(\mathbf{e})$. Error bars reflect mean \pm s.e.m. 
a
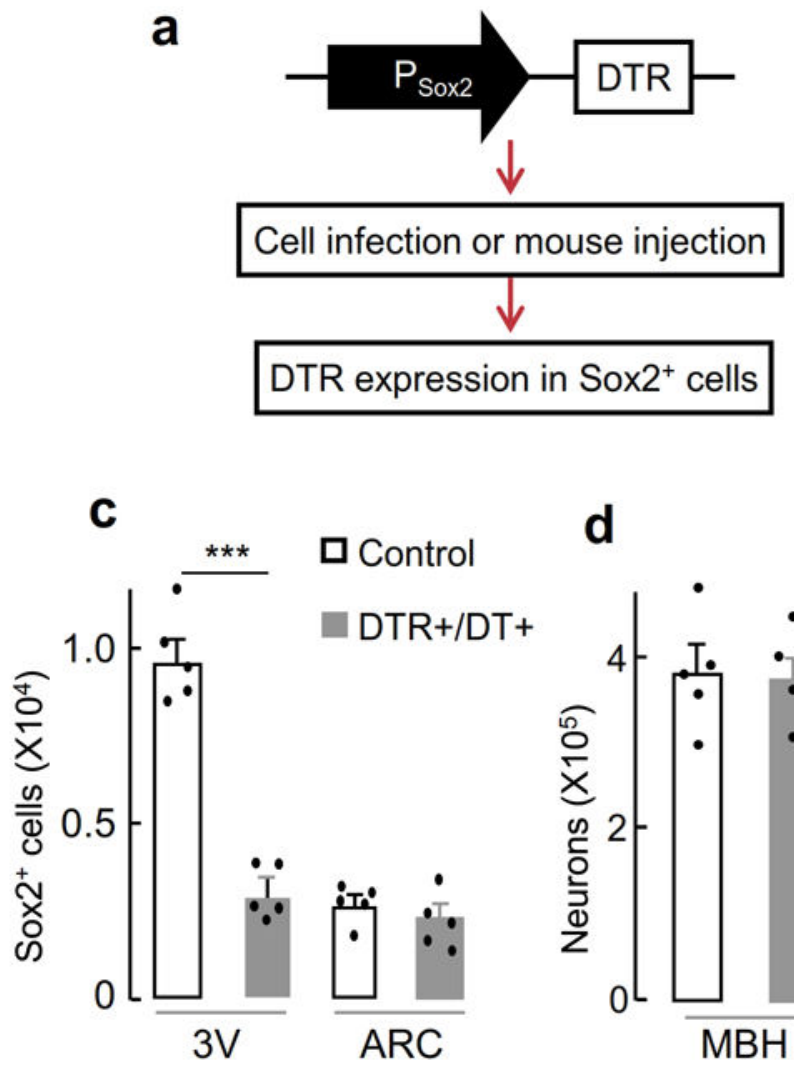

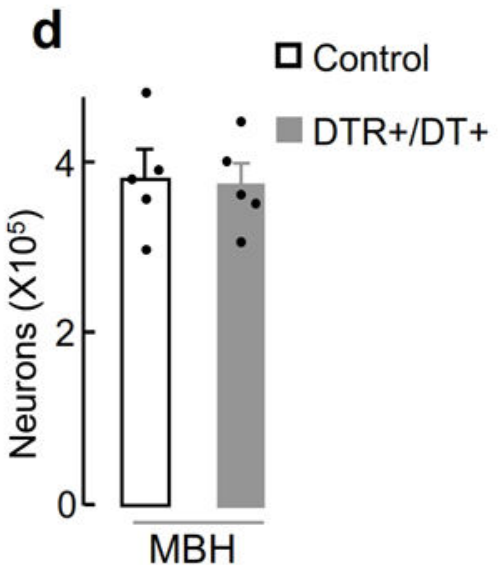

b
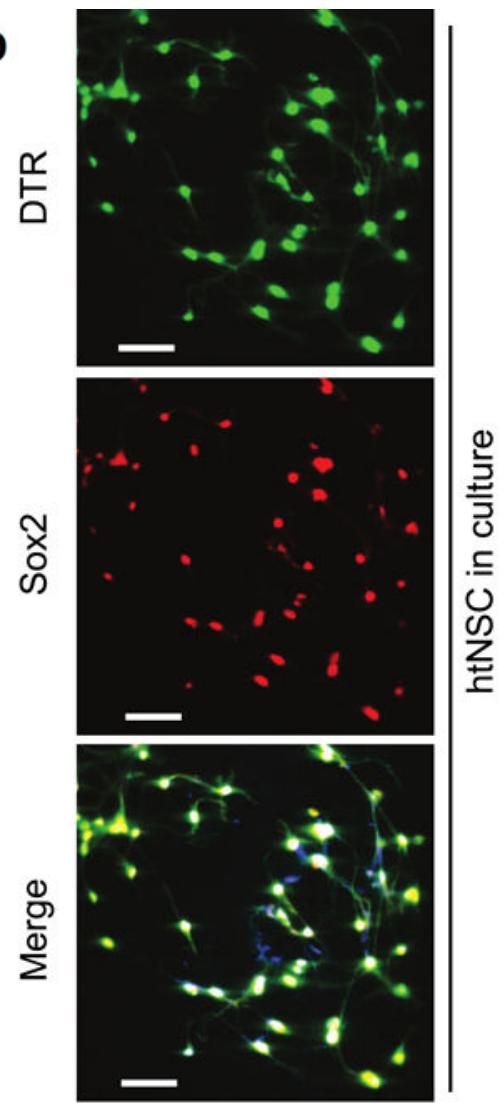
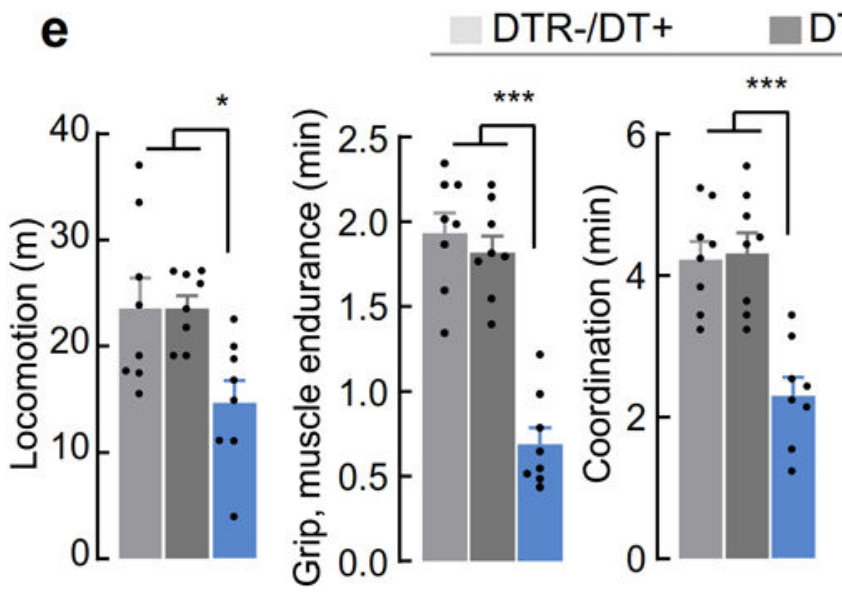

DTR+/DT-

DTR+/DT+
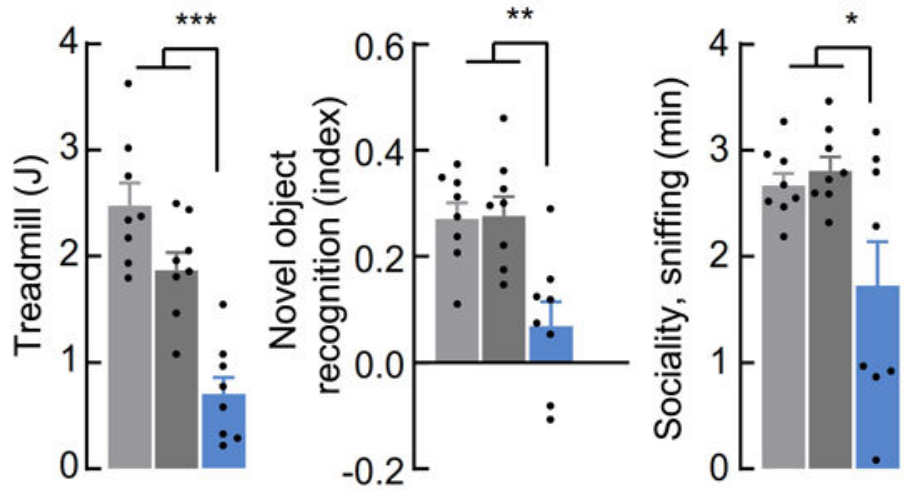

Extended Data Figure 3. Ablation of htNSC in hypothalamic 3V wall by DTR/DT

Mid-aged male C57BL/6 mice ( 15-month-old) were injected in the hypothalamic 3V with Sox2 promoter-directed DTR lentivirus (DTR) or control lentivirus, followed by 4-week i.p. injection of DT or vehicle. a, Diagram of lentiviral DTR. b, Evaluation on Sox 2 promoterdriven DTR lentiviruses in cultured htNSC via immunostaining for DTR and Sox2. Scale bar, $50 \mu \mathrm{m}$. c-e, Immunostaining of hypothalamic sections (c, d) and physiological analyses (e) of these mice at 3 months post viral injection. Control values in $\mathbf{c}$ and $\mathbf{d}$ represent similar observations in DTR-/DT+ and DTR+/DT- groups. Images represent 3 independent 
experiments (b). ${ }^{*} p<0.05, * * p<0.01, * * * p<0.001$, two-tailed Student's t-test (c, d), one-way ANOVA with Tukey's post-hoc test $(\mathbf{e}) ; \mathrm{n}=5$ mice per group $(\mathbf{c}, \mathbf{d})$ and $\mathrm{n}=8$ mice per group (e). Error bars reflect mean \pm s.e.m.
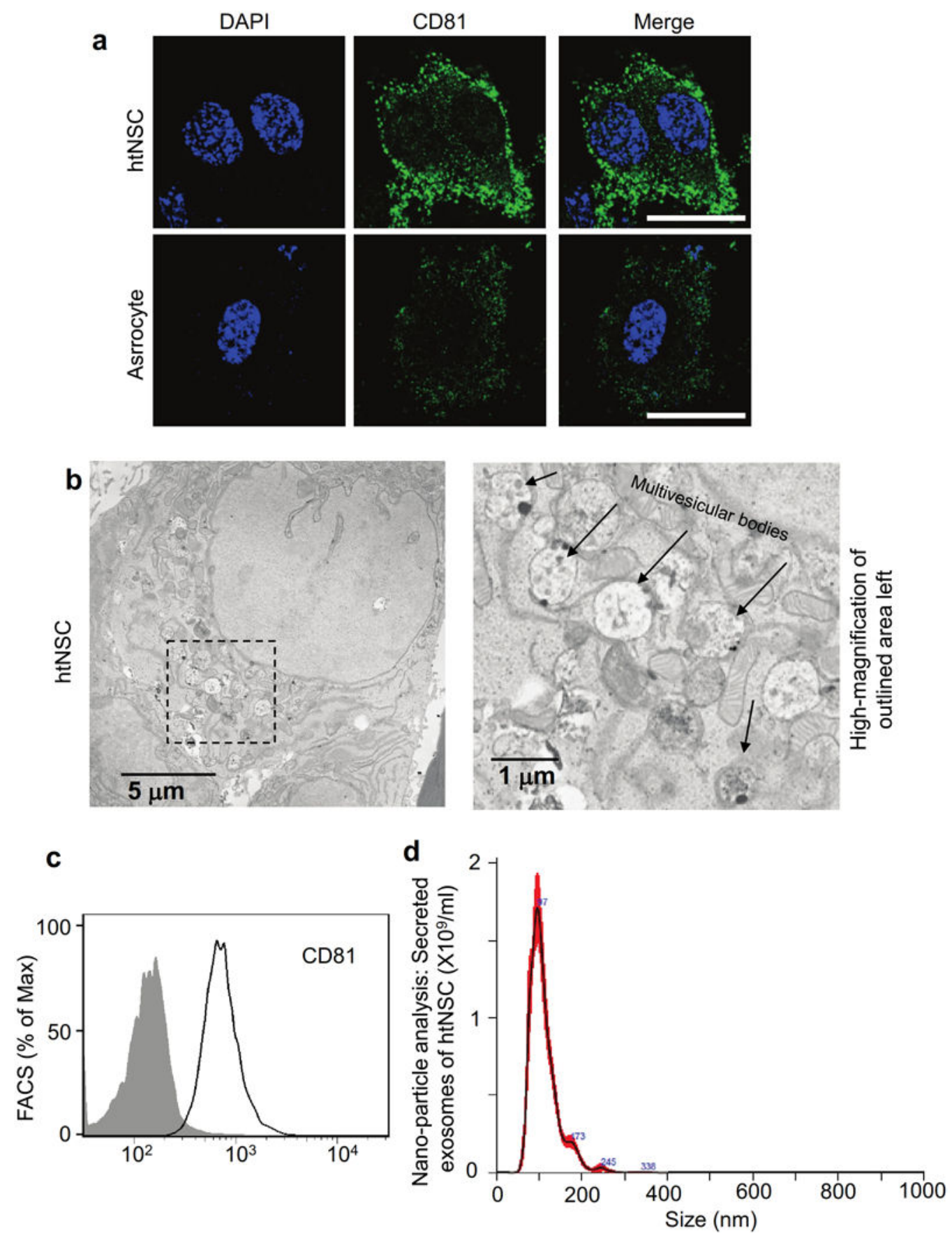

Extended Data Figure 4. Assessing exosomes secreted by hypothalamic NSC

a, Immunostaining of CD81 in cultured htNSC and astrocytes. Scale bar, $10 \mu \mathrm{m}$. b, Electron microscopic images of hypothalamic NSC (htNSC). Right panel shows high magnification of the outlined area in the left panel. Black arrows indicate the presence of multivescular 
bodies. c, Flow cytometry analysis of CD81 of hypothalamic NSC-derived secretory exosomes. Grey areas are appropriate isotype control. d, Purified exosomes secreted from cultured hypothalamic NSC were profiled using nano-particle analysis. Data represent 3 independent experiments.
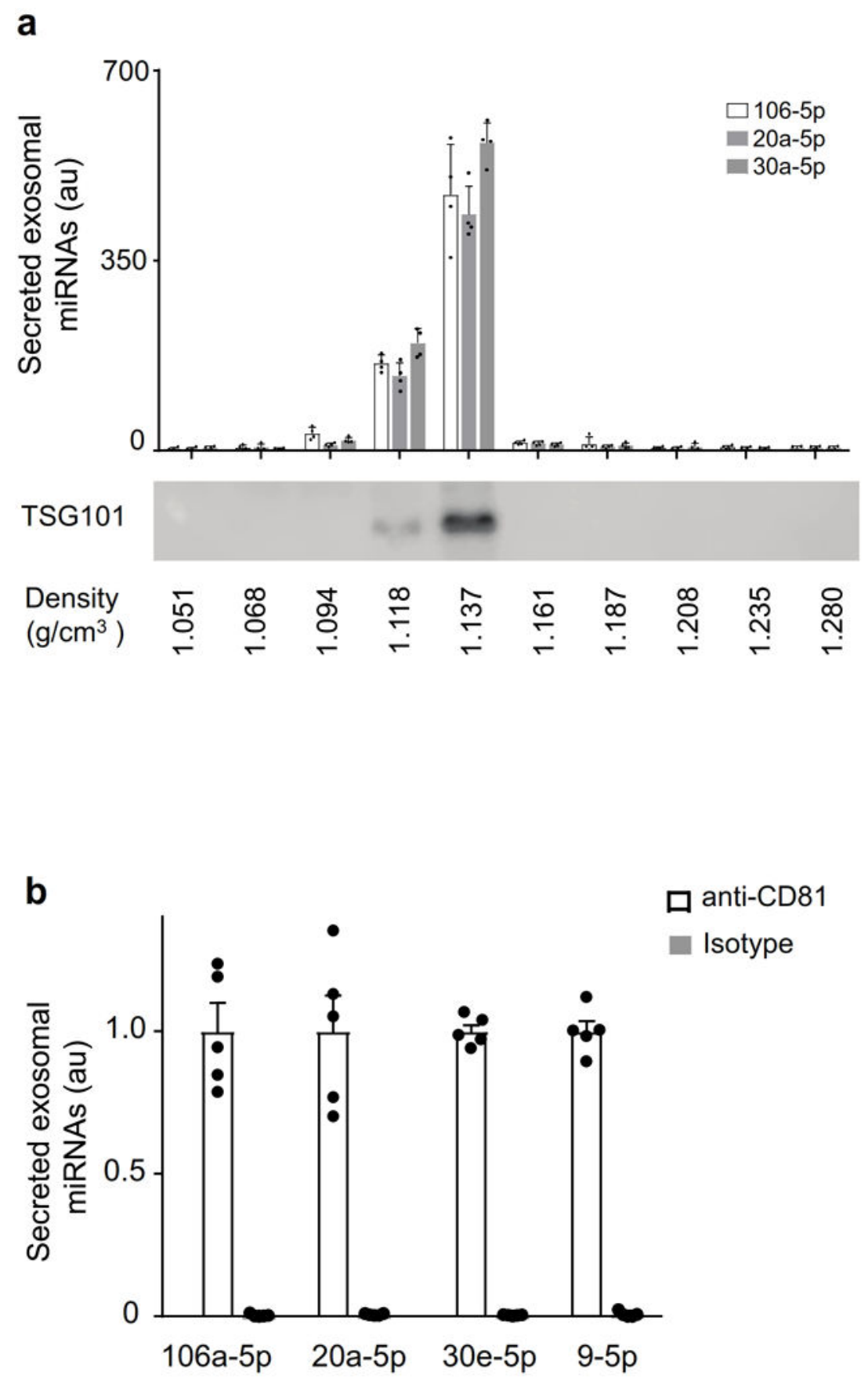

Extended Data Figure 5. Additional assessments on secreted exosomes from htNSC

a, Secreted exosomes isolated from htNSC were subjected to fractioning by density gradient ultracentrifuge, different fractions were analyzed by immunoblotting with anti-TSG101

Nature. Author manuscript; available in PMC 2018 June 13. 
antibody. b, Secretory exosomes isolated from htNSC were subjected to pull-down via antiCD81 antibody vs. appropriate isotype and then analyzed for levels of candidate miRNAs. $n$ $=4$ independent biological samples per group (a) and $n=5$ independent biological samples per group (b). Error bars reflect mean \pm s.e.m.

a
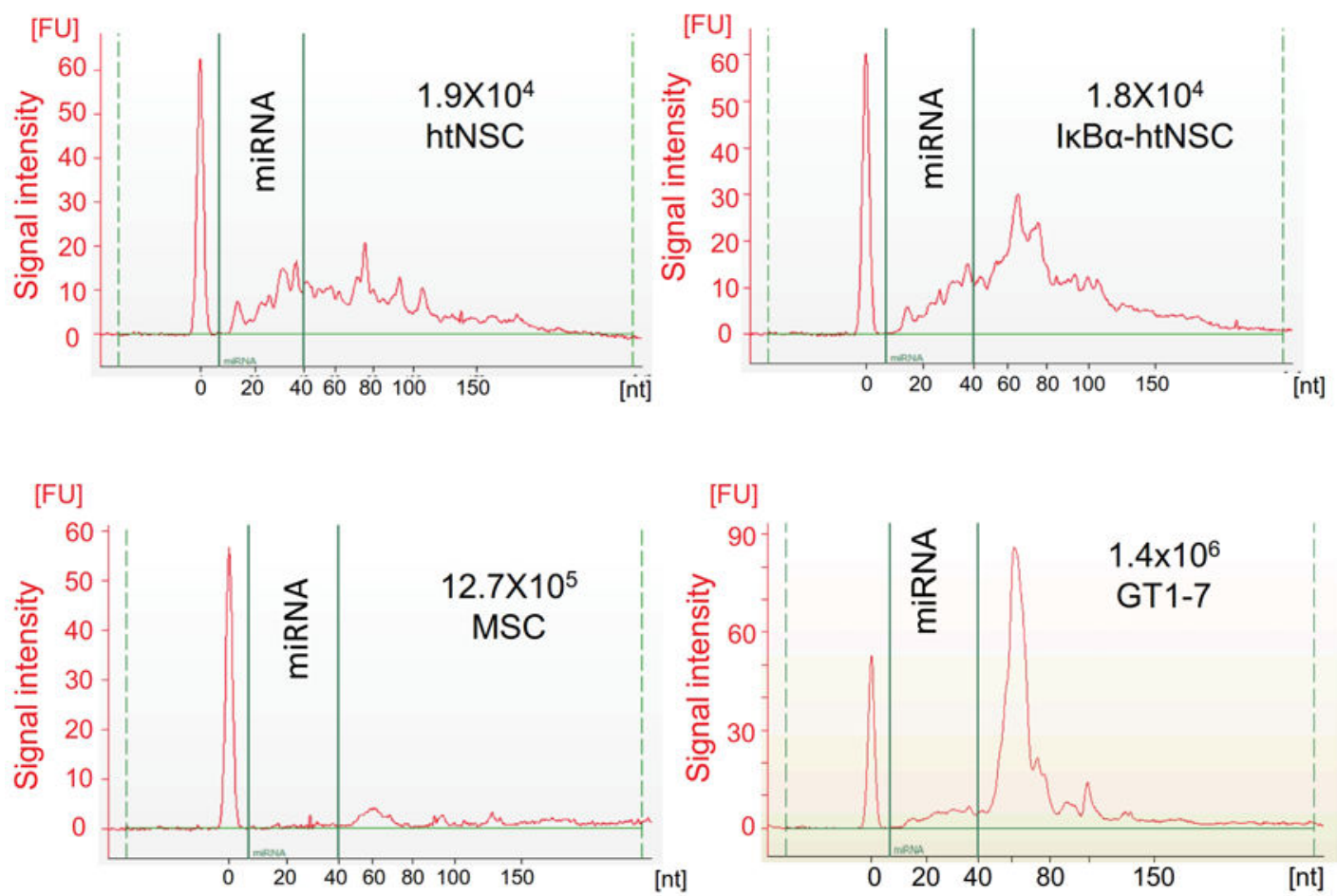

b
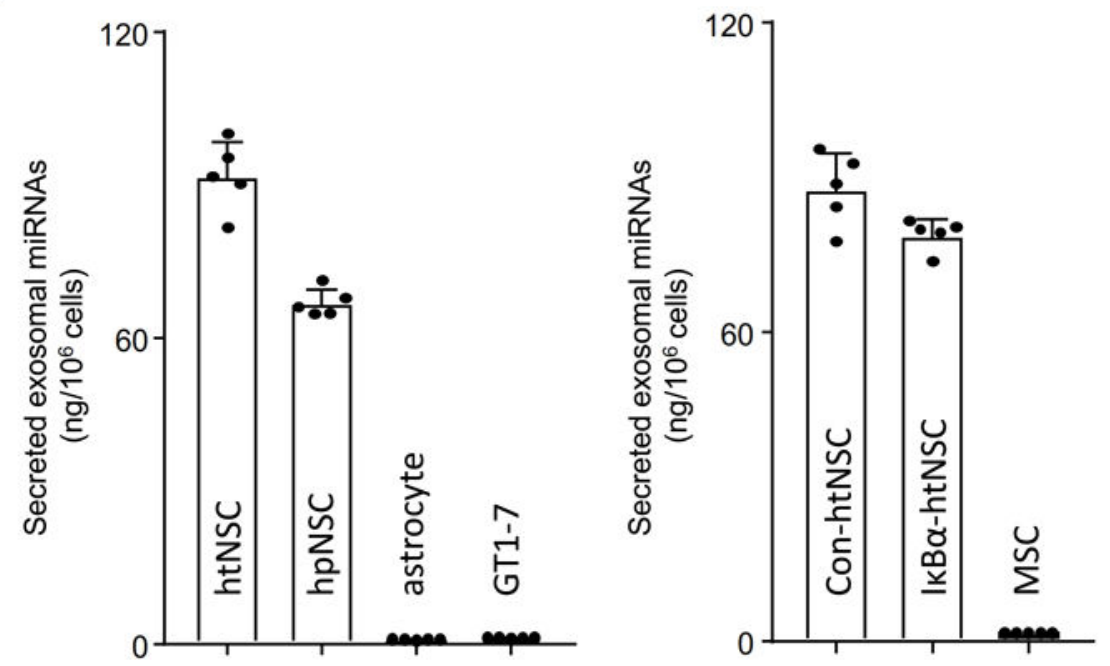

Extended Data Figure 6. Small RNA bio-analyzer assay of purified secretory exosomes a, Representative tracings of exosomal small RNA and miRNA secreted by htNSC, I $k \mathrm{Ba}$ htNSC, mesenchymal stem cells (MSC) and hypothalamic neuronal line GT1-7 cells. b. 
Quantitation of exosomal miRNAs secreted by indicated cells according to the results from small RNA/miRNA bio-analysis. $\mathrm{n}=5$ independent biological samples per group. Error bars reflect mean \pm s.e.m.

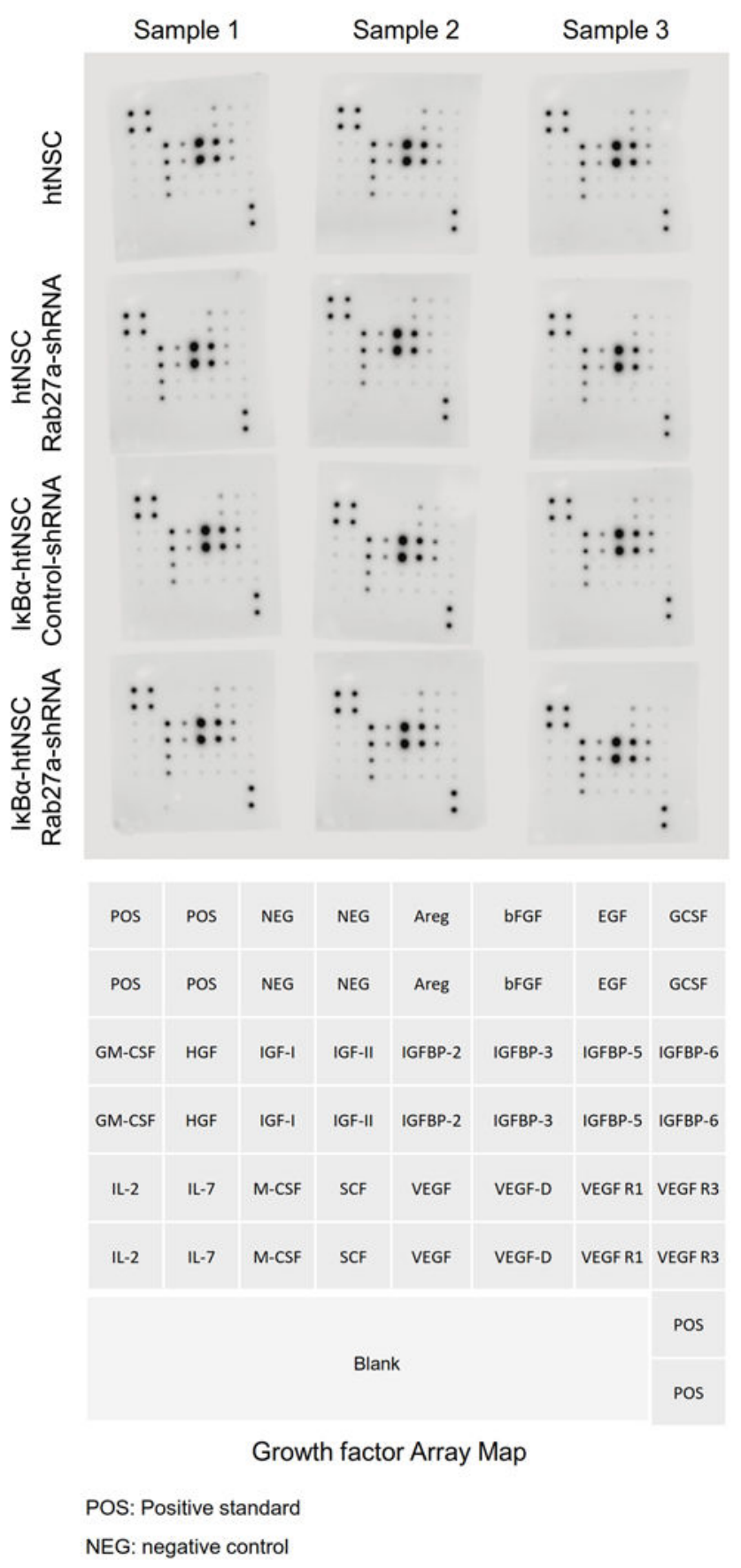

Extended Data Figure 7. Growth factors and cytokines secreted by htNSC

Indicated cells were cultured in media without containing EGF and bFGF for 2 days, and media were collected and analyzed using Mouse Growth Factor Array for indicated growth 
factors and cytokines. Array images contain 3 independent biological samples per cell model (blots on the top while the design of array on the bottom).
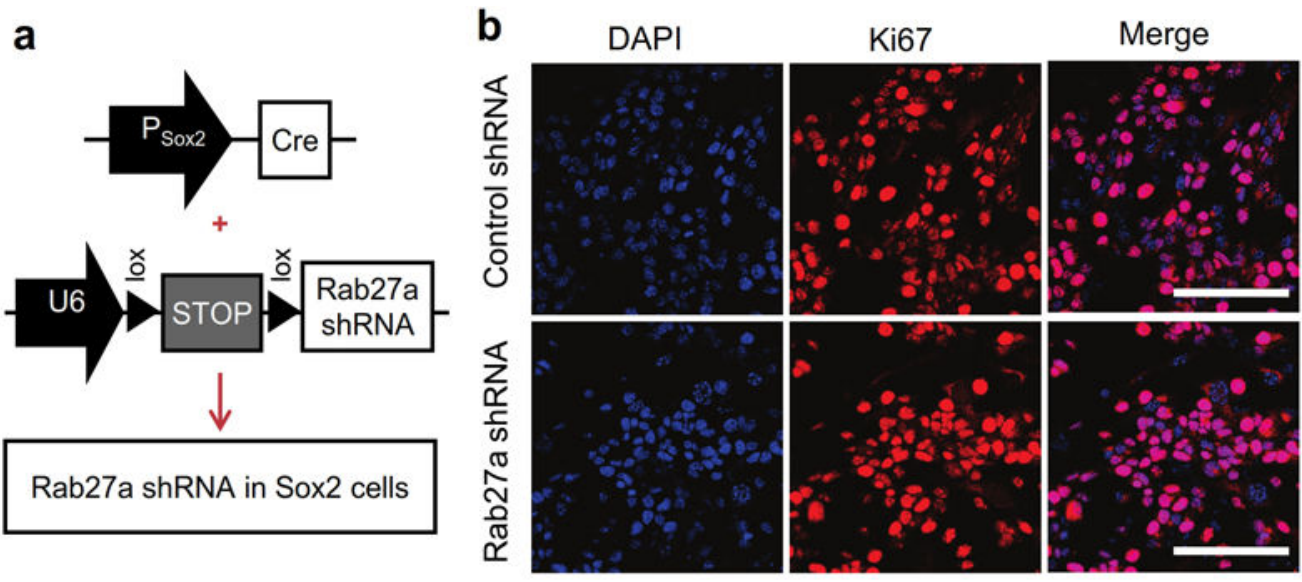

C

Vehicle

Control shRNA

Rab27a shRNA
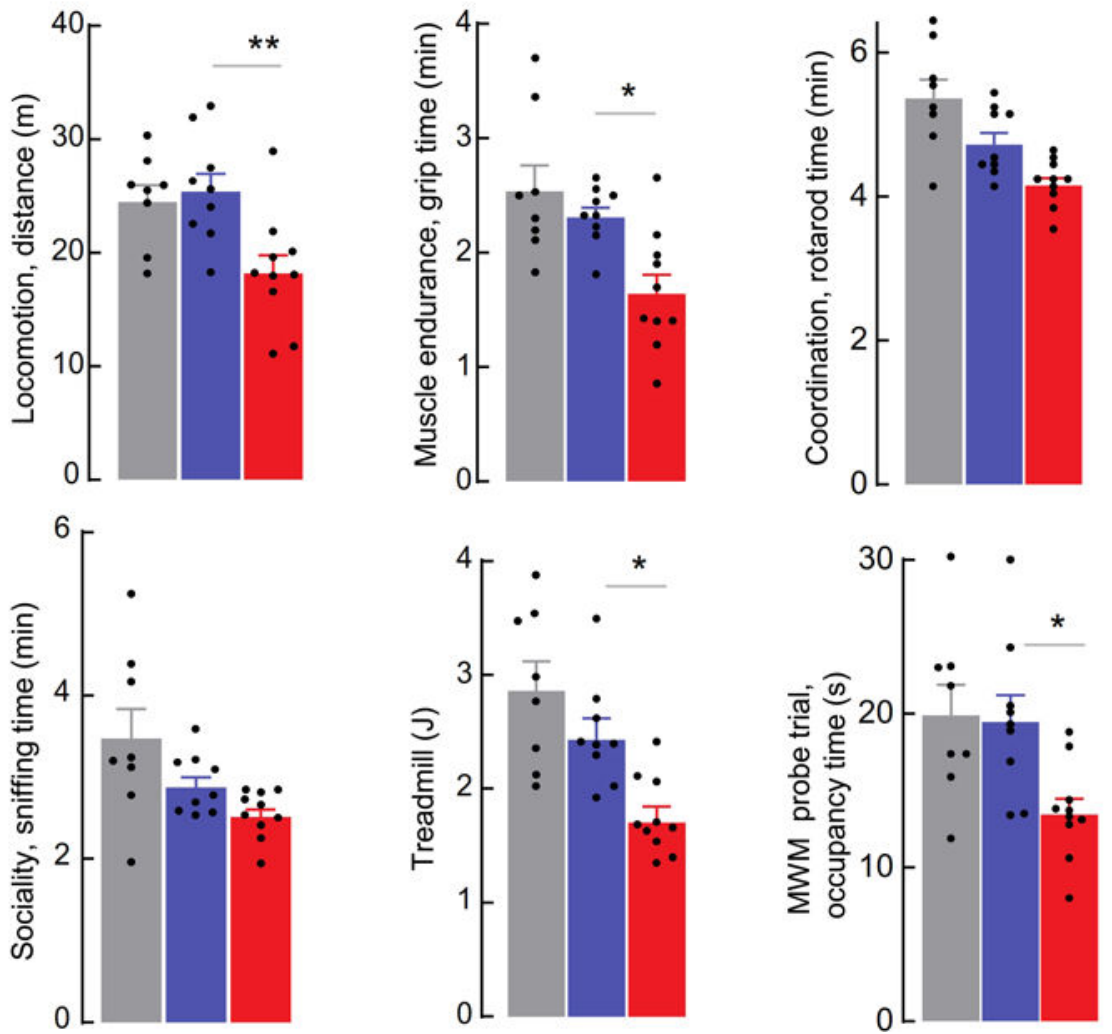

Extended Data Figure 8. Effects on htNSC and animal physiology by Rab27a shRNA a, b, Cultured htNSC were infected with Sox 2 promoter-driven Cre lentivirus and Credependent Rab27a shRNA vs. control scramble shRNA lentivirus (a) and examined for Ki67 via immunostaining (b) at 2-3 days post viral infection (Scale bars, $60 \mu \mathrm{m}$ ). c, C57BL/6 mice (12-month-old males) were injected in the hypothalamic $3 \mathrm{~V}$ with Sox 2 promoter- 
driven Cre lentivirus and Cre-dependent Rab27a shRNA vs. control scramble shRNA lentivirus or vehicle. Aging-related physiology was analyzed in mice at 6 weeks post viral injection. $* p<0.05, * * p<0.01$, one-way ANOVA with Tukey's post-hoc test (c); $\mathrm{n}=8$ mice for Vehicle, $\mathrm{n}=9$ mice for Control shRNA, $\mathrm{n}=10$ mice for Rab27a shRNA (c). Error bars reflect mean \pm s.e.m.

\section{Purfied htNSC-secreted exosomes and size distribution}

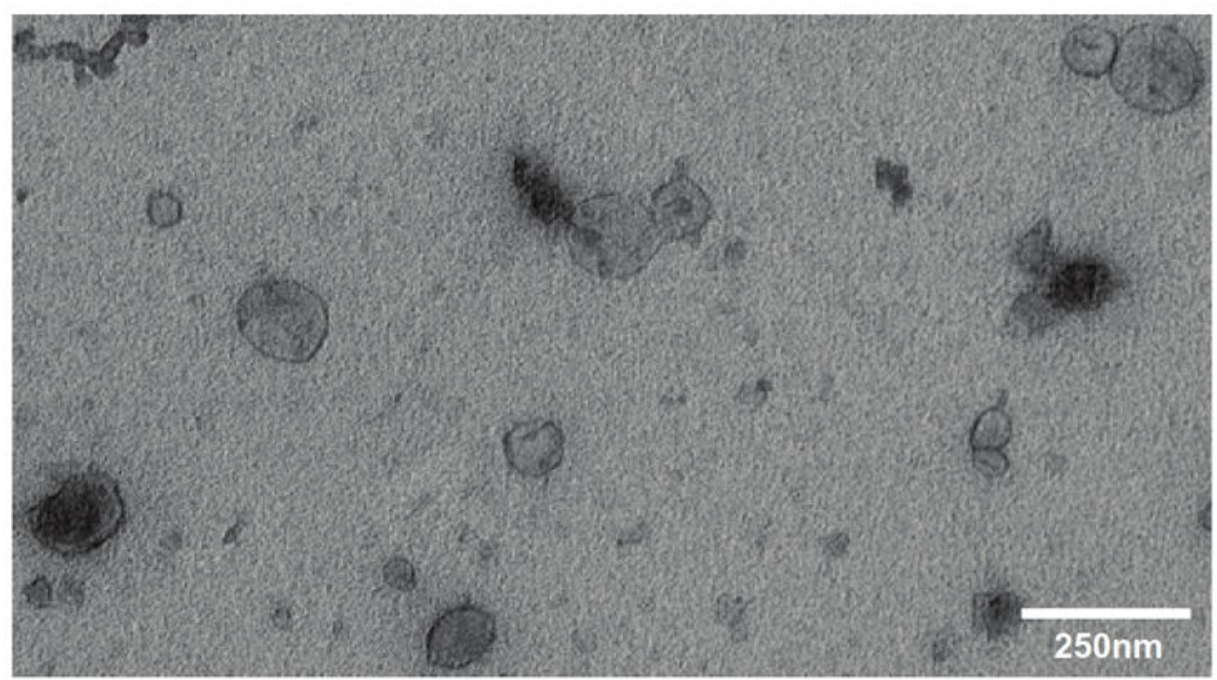

Extended Data Figure 9. Electron microscopic examination of htNSC-secreted exosomes Exosomes secreted from cultured htNSC were obtained and purified using differential ultracentrifugation and examined for the purity and size distribution via electron microscope using the protocol as detailed in Method section. Scale bar, $250 \mathrm{~nm}$. 

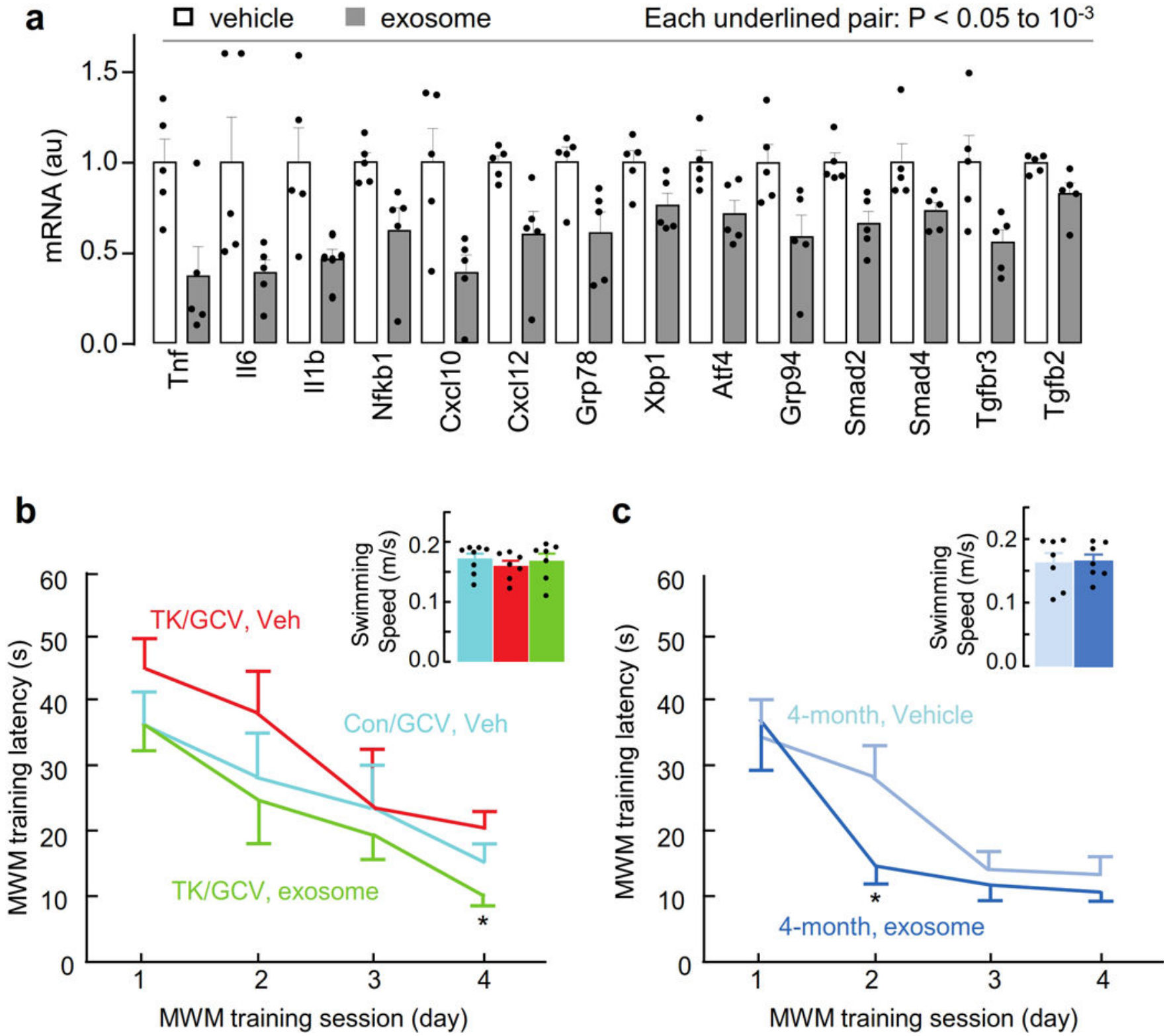

Extended Data Figure 10. Additional information on the anti-aging models of this study

a, C57BL/6 mice (16-month-old males) were treated via hypothalamic 3V cannula with exosomes (100 ng protein, purified from htNSC) vs. vehicle, 3 times per week for 4 months, and the hypothalamus tissues were dissected and examined for indicated mRNAs.

Expression levels of mRNAs were presented in arbitrary unit (au), and the value of each species in control group was normalized as 1 . $\mathbf{b}$, The training session information for MWM in Fig. 6c. c, The training session information for MWM in Fig. 6d. ${ }^{*} p<0.05$ (c) or per indication (a), two-tailed Student's t-test (a) or one-way ANOVA with Tukey's post-hoc test (c); $\mathrm{n}=5$ mice per group (a), $\mathrm{n}=8$ mice for Con/GCV with vehicle, $\mathrm{n}=7$ mice for TK/GCV with vehicle and TK/GCV with exosome (b), and $n=7$ mice per group (c). Error bars reflect mean \pm s.e.m. 


\section{Supplementary Material}

Refer to Web version on PubMed Central for supplementary material.

\section{Acknowledgments}

This study was supported by NIH R01 DK078750, AG031774, HL113180 and DK099136 (D. Cai).

\section{References}

1. Chang HC, Guarente L. SIRT1 Mediates Central Circadian Control in the SCN by a Mechanism that Decays with Aging. Cell. 2013; 153:1448-1460. DOI: 10.1016/j.cell.2013.05.027 [PubMed: 23791176]

2. Fridell YW, Sanchez-Blanco A, Silvia BA, Helfand SL. Targeted expression of the human uncoupling protein 2 (hUCP2) to adult neurons extends life span in the fly. Cell Metab. 2005; 1:145-152. DOI: 10.1016/j.cmet.2005.01.005 [PubMed: 16054055]

3. Alcedo J, Kenyon C. Regulation of C. elegans longevity by specific gustatory and olfactory neurons. Neuron. 2004; 41:45-55. [PubMed: 14715134]

4. Riera CE, et al. TRPV1 pain receptors regulate longevity and metabolism by neuropeptide signaling. Cell. 2014; 157:1023-1036. DOI: 10.1016/j.cell.2014.03.051 [PubMed: 24855942]

5. Zhang G, et al. Hypothalamic programming of systemic ageing involving IKK-beta, NF-kappaB and GnRH. Nature. 2013; 497:211-216. DOI: 10.1038/nature12143 [PubMed: 23636330]

6. Satoh A, et al. Sirt1 extends life span and delays aging in mice through the regulation of Nk2 homeobox 1 in the DMH and LH. Cell Metab. 2013; 18:416-430. DOI: 10.1016/j.cmet.2013.07.013 [PubMed: 24011076]

7. Dacks PA, Moreno CL, Kim ES, Marcellino BK, Mobbs CV. Role of the hypothalamus in mediating protective effects of dietary restriction during aging. Frontiers in neuroendocrinology. 2013; 34:95106. DOI: 10.1016/j.yfrne.2012.12.001 [PubMed: 23262258]

8. Sadagurski M, et al. Transient early food restriction leads to hypothalamic changes in the long-lived crowded litter female mice. Physiol Rep. 2015; 3

9. van Praag H, et al. Functional neurogenesis in the adult hippocampus. Nature. 2002; 415:10301034. DOI: $10.1038 / 4151030 a$ [PubMed: 11875571$]$

10. Encinas JM, et al. Division-coupled astrocytic differentiation and age-related depletion of neural stem cells in the adult hippocampus. Cell Stem Cell. 2011; 8:566-579. DOI: 10.1016/j.stem. 2011.03.010 [PubMed: 21549330]

11. Kheirbek MA, Klemenhagen KC, Sahay A, Hen R. Neurogenesis and generalization: a new approach to stratify and treat anxiety disorders. Nat Neurosci. 2012; 15:1613-1620. DOI: 10.1038/nn.3262 [PubMed: 23187693]

12. Merkle FT, et al. Adult neural stem cells in distinct microdomains generate previously unknown interneuron types. Nat Neurosci. 2014; 17:207-214. DOI: 10.1038/nn.3610 [PubMed: 24362763]

13. Sun Y, et al. Neurogenin promotes neurogenesis and inhibits glial differentiation by independent mechanisms. Cell. 2001; 104:365-376. [PubMed: 11239394]

14. Molofsky AV, et al. Increasing p16INK4a expression decreases forebrain progenitors and neurogenesis during ageing. Nature. 2006; 443:448-452. DOI: 10.1038/nature05091 [PubMed: 16957738]

15. Baruch K, et al. Aging Aging-induced type I interferon response at the choroid plexus negatively affects brain function. Science. 2014; 346:89-93. DOI: 10.1126/science.1252945 [PubMed: 25147279]

16. Greenberg DA, Jin K. Turning neurogenesis up a Notch. Nat Med. 2006; 12:884-885. DOI: 10.1038/nm0806-884 [PubMed: 16892029]

17. Villeda SA, et al. The ageing systemic milieu negatively regulates neurogenesis and cognitive function. Nature. 2011; 477:90-94. DOI: 10.1038/nature10357 [PubMed: 21886162] 
18. Sun F, et al. Notch1 signaling modulates neuronal progenitor activity in the subventricular zone in response to aging and focal ischemia. Aging Cell. 2013; 12:978-987. DOI: 10.1111/acel.12134 [PubMed: 23834718]

19. Li J, Tang Y, Cai D. IKKbeta/NF-kappaB disrupts adult hypothalamic neural stem cells to mediate a neurodegenerative mechanism of dietary obesity and pre-diabetes. Nat Cell Biol. 2012; 14:999_ 1012. doi:ncb2562 [pii];10.1038/ncb2562 [doi]. [PubMed: 22940906]

20. Lee DA, et al. Tanycytes of the hypothalamic median eminence form a diet-responsive neurogenic niche. Nat Neurosci. 2012; 15:700-702. DOI: 10.1038/nn.3079 [PubMed: 22446882]

21. McNay DE, Briancon N, Kokoeva MV, Maratos-Flier E, Flier JS. Remodeling of the arcuate nucleus energy-balance circuit is inhibited in obese mice. J Clin Invest. 2012; 122:142-152. DOI: 10.1172/JCI43134 [PubMed: 22201680]

22. Favaro R, et al. Hippocampal development and neural stem cell maintenance require Sox2dependent regulation of Shh. Nat Neurosci. 2009; 12:1248-1256. DOI: 10.1038/nn.2397 [PubMed: 19734891]

23. Molofsky AV, et al. Bmi-1 dependence distinguishes neural stem cell self-renewal from progenitor proliferation. Nature. 2003; 425:962-967. DOI: 10.1038/nature02060 [PubMed: 14574365]

24. Strojnik T, Rosland GV, Sakariassen PO, Kavalar R, Lah T. Neural stem cell markers, nestin and musashi proteins, in the progression of human glioma: correlation of nestin with prognosis of patient survival. Surgical neurology. 2007; 68:133-143. discussion 143-134. DOI: 10.1016/ j.surneu.2006.10.050 [PubMed: 17537489]

25. Faiz M, et al. Adult Neural Stem Cells from the Subventricular Zone Give Rise to Reactive Astrocytes in the Cortex after Stroke. Cell Stem Cell. 2015; 17:624-634. DOI: 10.1016/j.stem. 2015.08.002 [PubMed: 26456685]

26. Corti S, et al. Neural stem cells LewisX+CXCR4+ modify disease progression in an amyotrophic lateral sclerosis model. Brain : a journal of neurology. 2007; 130:1289-1305. DOI: 10.1093/brain/ awm043 [PubMed: 17439986]

27. Buch T, et al. A Cre-inducible diphtheria toxin receptor mediates cell lineage ablation after toxin administration. Nat Methods. 2005; 2:419-426. DOI: 10.1038/nmeth762 [PubMed: 15908920]

28. Shi Y, et al. MicroRNA regulation of neural stem cells and neurogenesis. J Neurosci. 2010; 30:14931-14936. DOI: 10.1523/JNEUROSCI.4280-10.2010 [PubMed: 21068294]

29. Li Q, Gregory RI. MicroRNA regulation of stem cell fate. Cell Stem Cell. 2008; 2:195-196. DOI: 10.1016/j.stem.2008.02.008 [PubMed: 18371442]

30. Boon RA, et al. MicroRNA-34a regulates cardiac ageing and function. Nature. 2013; 495:107-110. DOI: 10.1038/nature11919 [PubMed: 23426265]

31. Fraczek LA, Martin CB, Martin BK. c-Jun and c-Fos regulate the complement factor H promoter in murine astrocytes. Mol Immunol. 2011; 49:201-210. DOI: 10.1016/j.molimm.2011.08.013 [PubMed: 21920606]

32. Zhang X, et al. Hypothalamic IKKbeta/NF-kappaB and ER stress link overnutrition to energy imbalance and obesity. Cell. 2008; 135:61-73. [PubMed: 18854155]

33. Soleimani M, Nadri S. A protocol for isolation and culture of mesenchymal stem cells from mouse bone marrow. Nat Protoc. 2009; 4:102-106. DOI: 10.1038/nprot.2008.221 [PubMed: 19131962]

34. Jin K, Wang X, Xie L, Mao XO, Greenberg DA. Transgenic ablation of doublecortin-expressing cells suppresses adult neurogenesis and worsens stroke outcome in mice. Proc Natl Acad Sci U S A. 2010; 107:7993-7998. DOI: 10.1073/pnas.1000154107 [PubMed: 20385829]

35. Li J, et al. Exosomes mediate the cell-to-cell transmission of IFN-alpha-induced antiviral activity. Nat Immunol. 2013; 14:793-803. DOI: 10.1038/ni.2647 [PubMed: 23832071]

36. Purkayastha S, Zhang G, Cai D. Uncoupling the mechanisms of obesity and hypertension by targeting hypothalamic IKK-beta and NF-kappaB. Nat Med. 2011; 17:883-887. DOI: 10.1038/nm. 2372 [PubMed: 21642978]

37. Fry CS, et al. Inducible depletion of satellite cells in adult, sedentary mice impairs muscle regenerative capacity without affecting sarcopenia. Nat Med. 2015; 21:76-80. DOI: 10.1038/nm. 3710 [PubMed: 25501907]

38. Kaidanovich-Beilin O, Lipina T, Vukobradovic I, Roder J, Woodgett JR. Assessment of social interaction behaviors. Journal of visualized experiments : JoVE. 2011 
39. Leger M, et al. Object recognition test in mice. Nat Protoc. 2013; 8:2531-2537. DOI: 10.1038/ nprot.2013.155 [PubMed: 24263092]

40. Yan J, et al. Obesity- and aging-induced excess of central transforming growth factor-beta potentiates diabetic development via an RNA stress response. Nat Med. 2014; 20:1001-1008. DOI: 10.1038/nm.3616 [PubMed: 25086906]

41. Aponte Y, Atasoy D, Sternson SM. AGRP neurons are sufficient to orchestrate feeding behavior rapidly and without training. Nat Neurosci. 2011; 14:351-355. DOI: 10.1038/nn.2739 [PubMed: 21209617] 

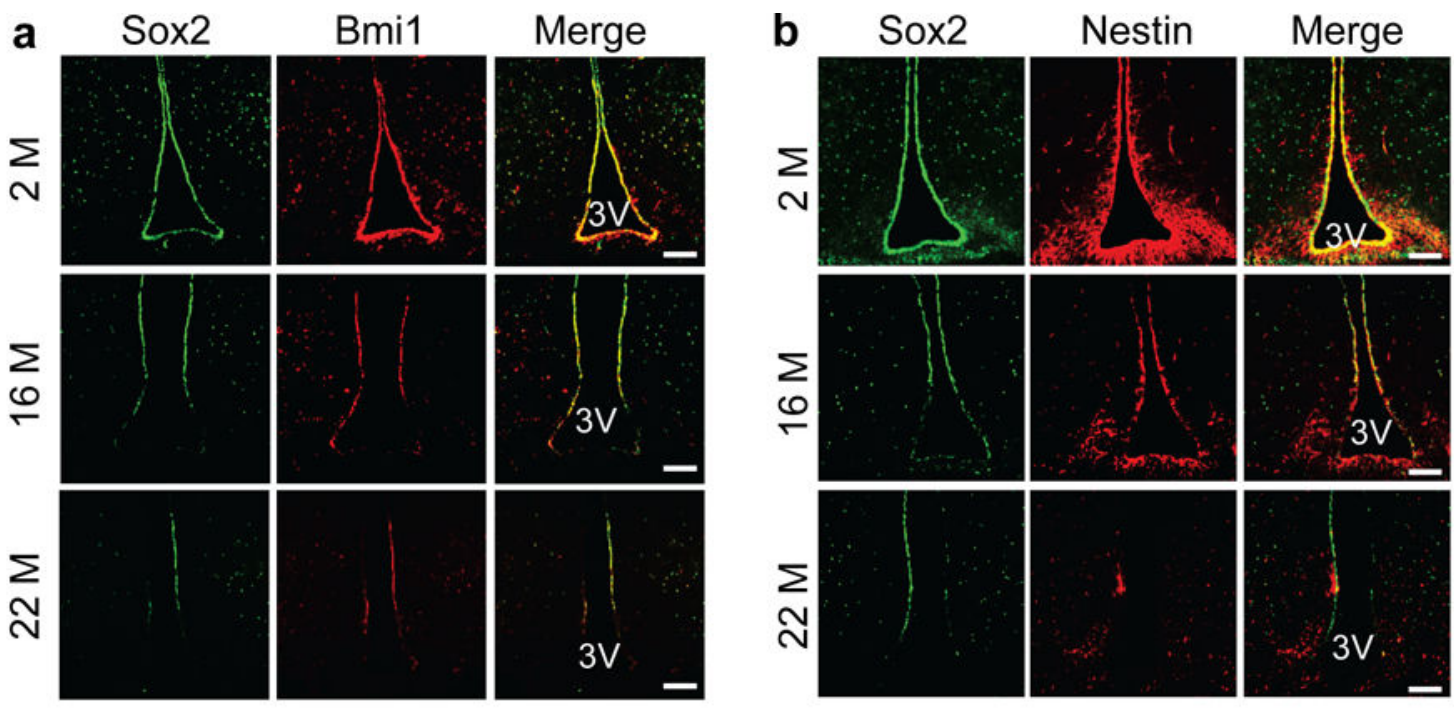

C

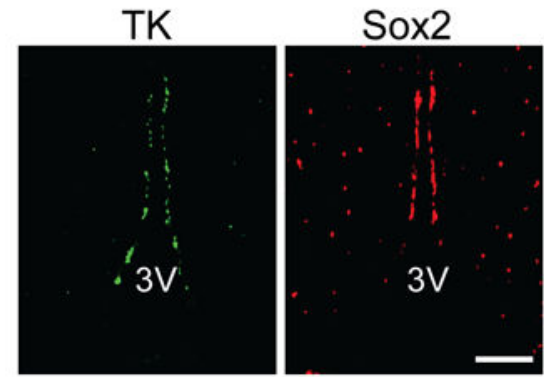

1-week post injection
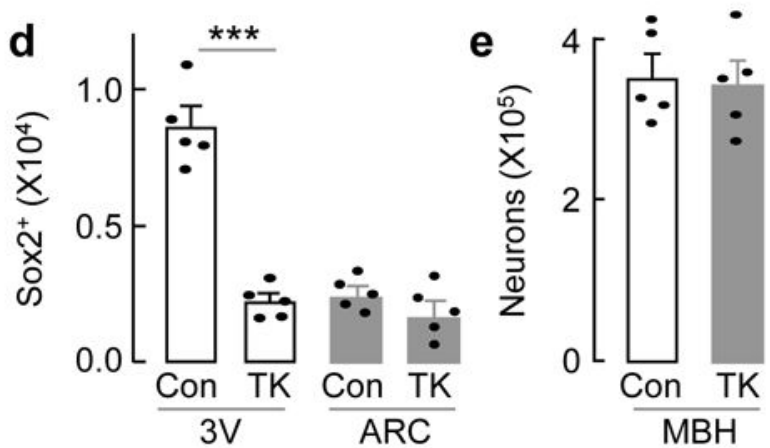

f $\quad \mathrm{TK}-/ \mathrm{GCV}+\quad \mathrm{TK}+/ \mathrm{GCV}-\quad \square \mathrm{TK}+/ \mathrm{GCV}+$
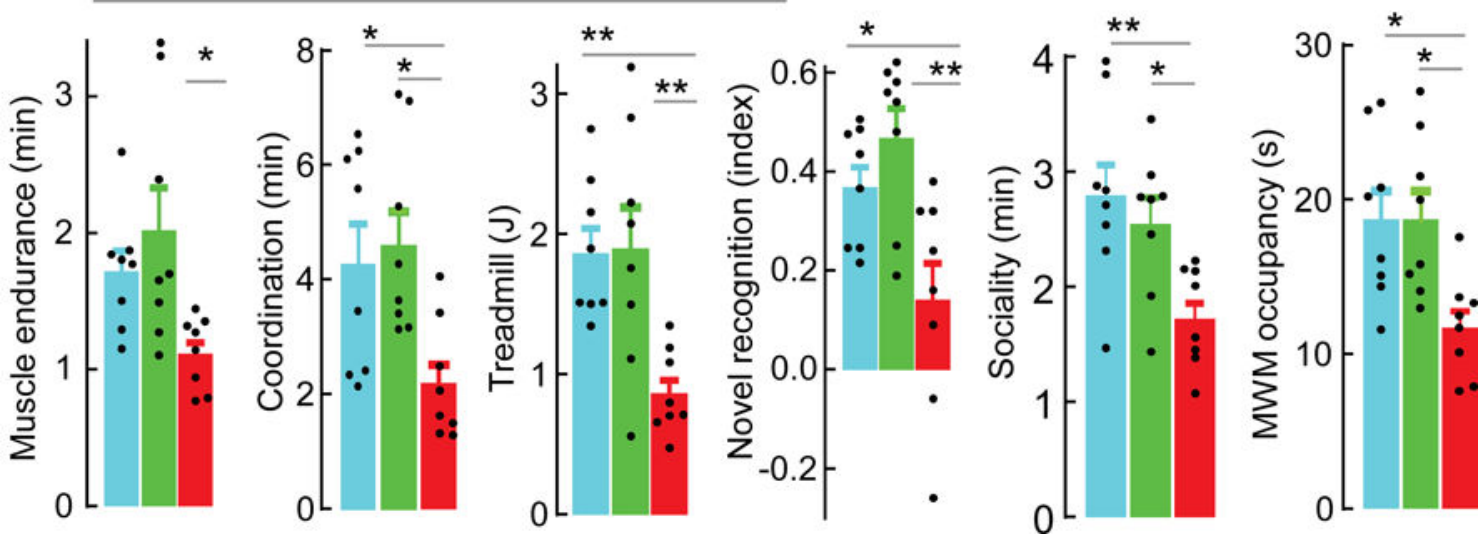

Figure 1. Aging-associated hypothalamic NSC loss and its impact on aging speed $\mathbf{a}, \mathbf{b}$, Hypothalamic sections from male C57BL/6 mice at indicated months (M) of age were immunostained for Sox2 (a, b) with Bmi1 (a) or Nestin (b). c-f, Mid-aged C57BL/6 mice ( 15-month-old) were injected in hypothalamic $3 \mathrm{~V}$ with Sox 2 promoter-directed Hsv-TK (TK) vs. control (Con) lentiviruses (c), followed by Ganciclovir (GCV) vs. vehicle treatment, and examined for cell ablation (d, e) and physiology (f) after 3 months post viral injection. d, e: Numbers of Sox2-postive cells and NeuN-positive neurons in hypothalamic 3V wall, arcuate nucleus (ARC) or MBH. MWM: Morris Water Maze (see ED Fig. 2e). 
Images represent 3 independent experiments (a-c), scale bar, $100 \mu \mathrm{m} . * p<0.05, * * p<$ 0.01 , *** $p<0.001$; two-tailed Student's t-test $(\mathbf{d}, \mathbf{e})$, one-way ANOVA with Tukey's test $(\mathbf{f}) ; \mathrm{n}=5$ mice $(\mathbf{d}, \mathbf{e})$ and $\mathrm{n}=8$ mice (f) per group. Error bars reflect mean \pm s.e.m. 
a

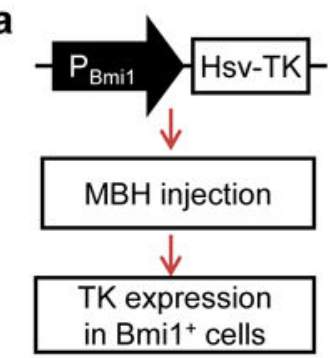
in Bmi1+ cells

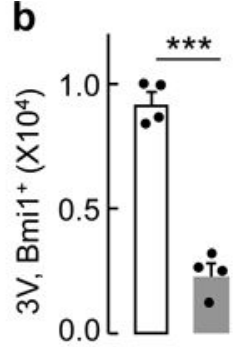

$\square$ Control $\square$ TK/GCV

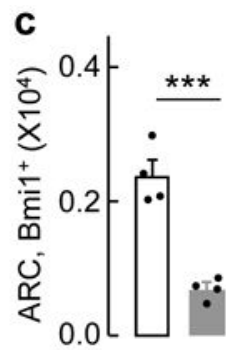

$\square$ Control $\square \mathrm{TK} / \mathrm{GCV}$

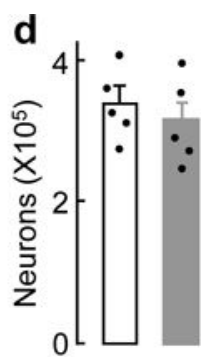

$\square$ Control $\square \mathrm{TK} / \mathrm{GCV}$
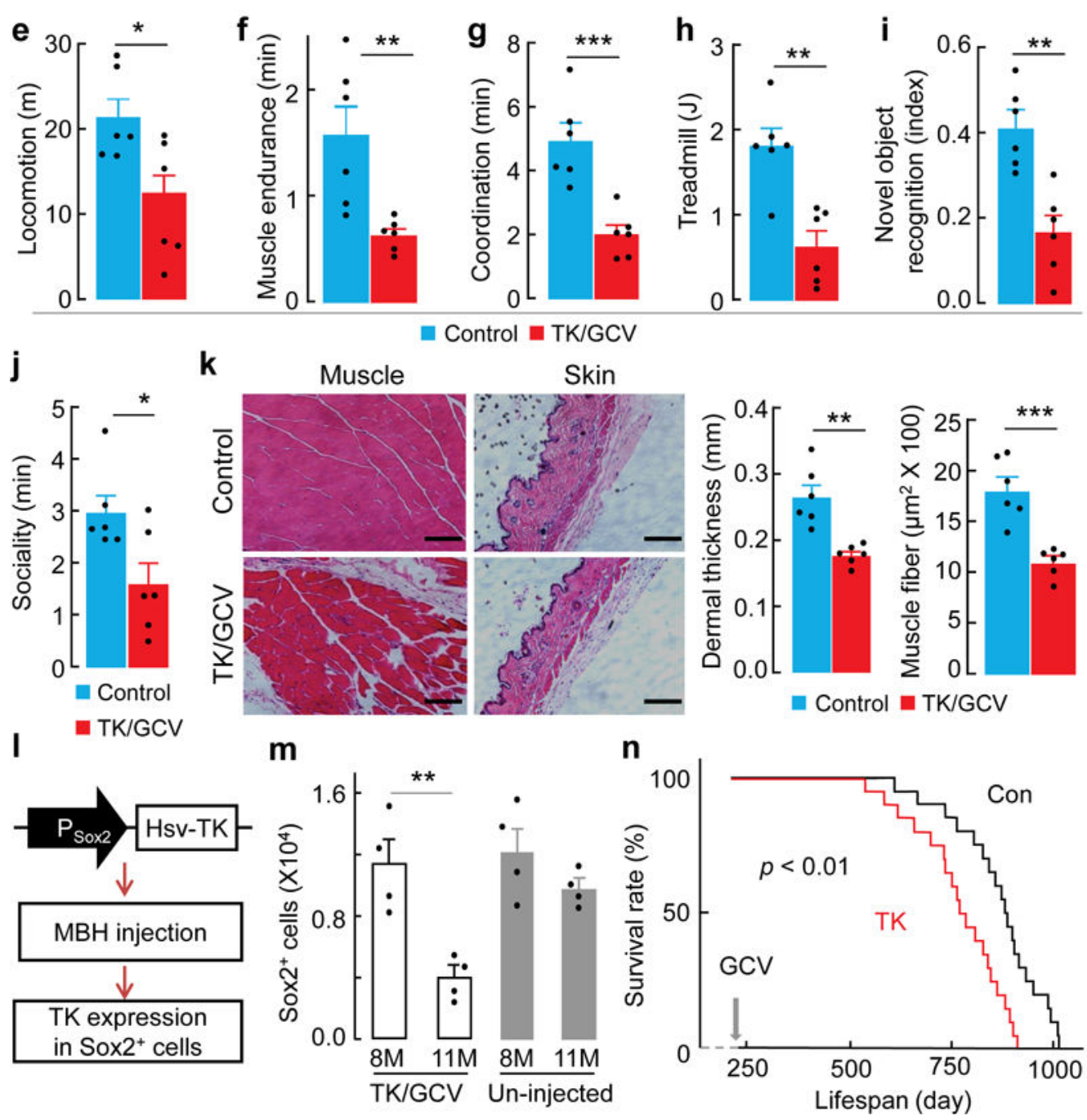

Figure 2. Aging acceleration and lifespan shortening due to hypothalamic NSC loss Mid-aged male C57BL/6 mice ( 15-month-old) were bilaterally injected in the MBH with Bmil promoter-driven Hsv-TK (TK) vs. control (Con) lentiviruses, followed by GCV vs. vehicle (Veh) treatment. a, Diagram of TK lentivirus and injection. b-d, Bmi1-positive cells in hypothalamic $3 \mathrm{~V}$ wall and $\mathrm{ARC}(\mathbf{b}, \mathbf{c})$ and $\mathrm{NeuN}$-positive neurons $(\mathbf{d})$ in the $\mathrm{MBH}$ at 3 months post viral injection. e-k, At 3 4 months post viral injection, TK+/GCV+ group (labeled as "TK/GCV") were compared to control groups (data derived from TK+/GCVgroup but also represented TK-/GCV+ and TK-/GCV-groups) for physiology (e-j) and 
histology (k). Scale bar, $100 \mu \mathrm{m}$. l-n, male C57BL/6 mice (8-month-old) were bilaterally injected in the MBH with Sox 2 promoter-driven Hsv-TK (TK) vs. control (Con) lentiviruses, both followed by GCV treatment. l, Diagram of TK lentivirus and injection. $\mathbf{m}$, Sox2-

positive cells in $\mathrm{MBH}$ parenchyma and hypothalamic $3 \mathrm{~V}$ wall of these mice (aged-matched un-injected mice included as a reference). $\mathbf{n}$, Lifespan follow-up. Images represent 2 independent experiments $(\mathbf{k}) * p<0.05$, ** $p<0.01$, *** $p<0.001$; two-tailed Student's ttest (b-k), two-way ANOVA with post-hoc test (m), log-rank (Mantel-Cox) test (n); $\mathrm{n}=4$ mice $(\mathbf{b}, \mathbf{c}, \mathbf{m})$ or $\mathrm{n}=5$ mice $(\mathbf{d})$ and $\mathrm{n}=6$ mice $(\mathbf{e}-\mathbf{k})$ per group, and $\mathrm{n}=19$ mice for Con and $\mathrm{n}=20$ mice for TK $(\mathbf{n})$. Error bars reflect mean \pm s.e.m. 
a
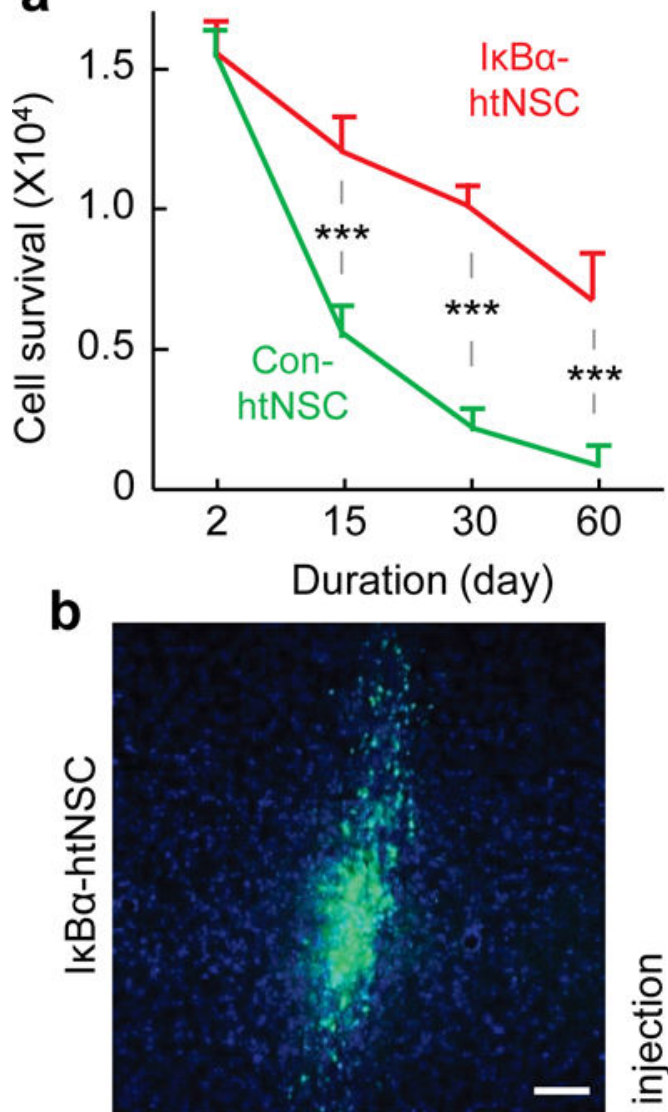

\section{Vehicle Astrocyte $\square$ MSC $\square$ IkBa-htNSC}
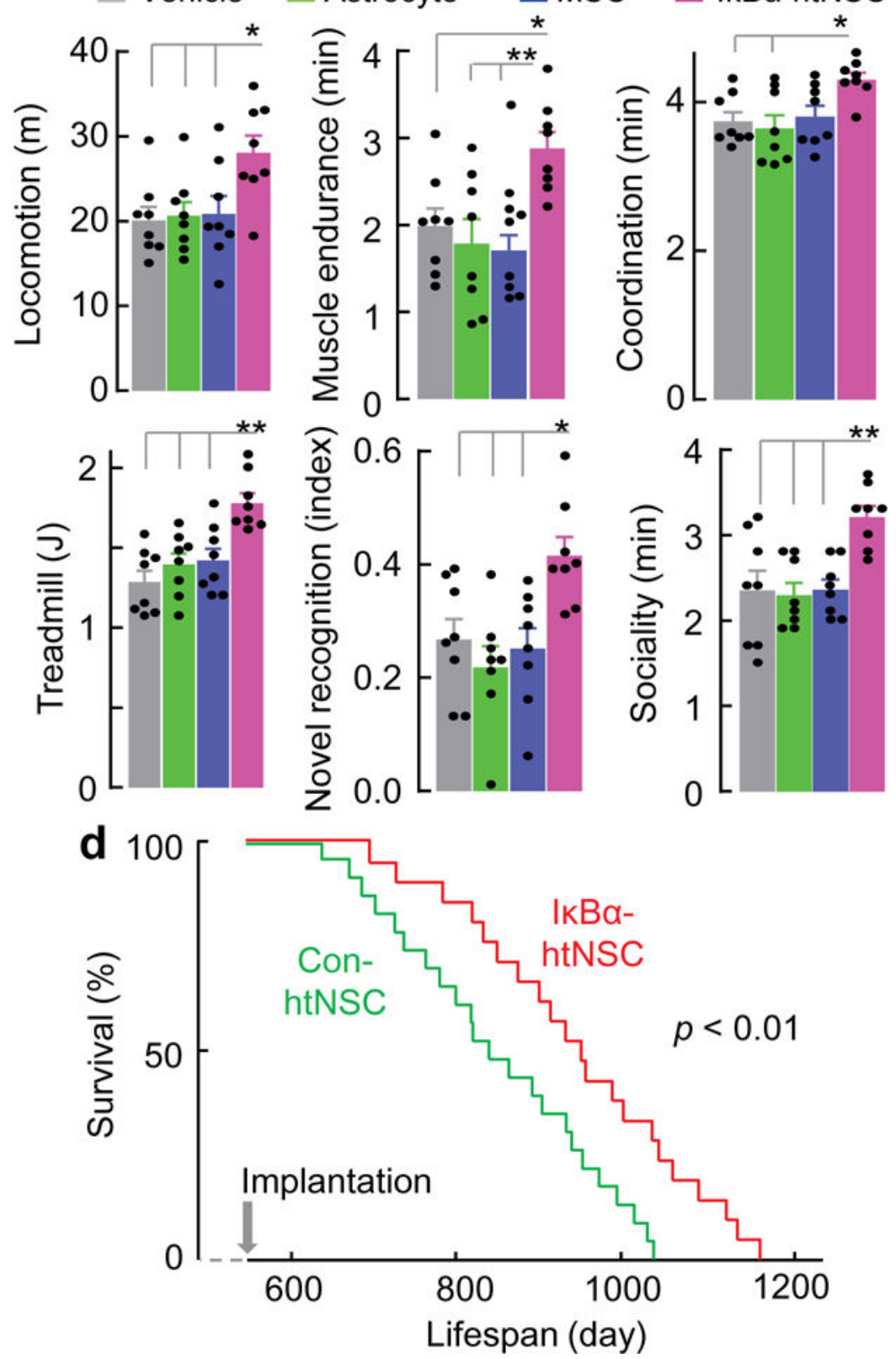

Figure 3. Slowdown of aging by hypothalamic implantation of IxBa-htNSC

a, GFP-expressing I $\mathrm{Ba}$-htNSC vs. Con-htNSC were implanted bilaterally into the $\mathrm{MBH}$ of mid-aged C57BL/6 mice and examined for survival at indicated days post implantation. b, c, GFP-labeled I $k \mathrm{Ba}$-htNSC and astrocytes as well as mesenchymal stem cells (MSC, image not shown) were implanted into the $\mathrm{MBH}$ of $\sim 16$-month-old male C57BL/6 mice and examined for GFP at 1 month post implantation (b) and aging-related physiology at 4 months post implantation (c). Scale bar, $100 \mu \mathrm{m}$. d, I $\mathrm{BBa}$-htNSC vs. Con-htNSC were implanted bilaterally into the $\mathrm{MBH}$ of male C57BL/6 mice (18-month-old) for lifespan follow-up. Images represent 2 independent experiments (b). ${ }^{*} p<0.05,{ }^{*} p<0.01$ (a, c); two-way ANOVA with post-hoc test (a), one-way ANOVA with Tukey's test (c), log-rank 
(Mantel-Cox) test (d); $\mathrm{n}=4$ mice (a) and $\mathrm{n}=8$ mice (c) per group, and $\mathrm{n}=23$ mice for ConhtNSC and $\mathrm{n}=21$ mice for I $k \mathrm{Ba}$-htNSC $(\mathbf{d})$. Error bars reflect mean \pm s.e.m. 
a

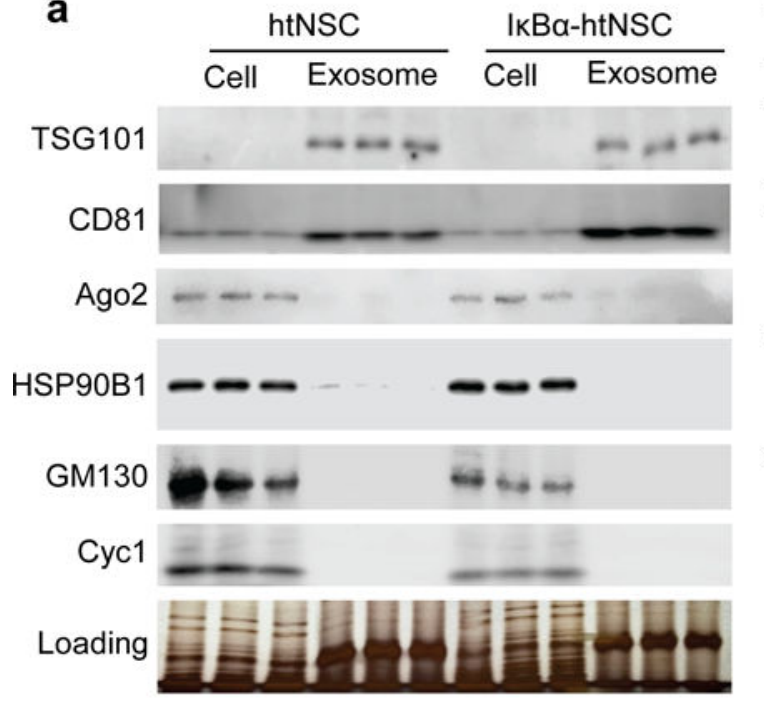

d

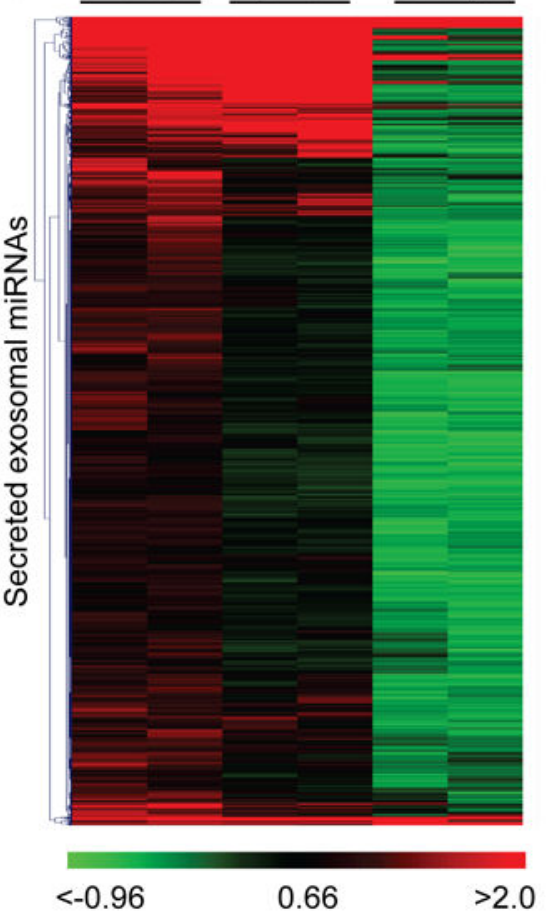

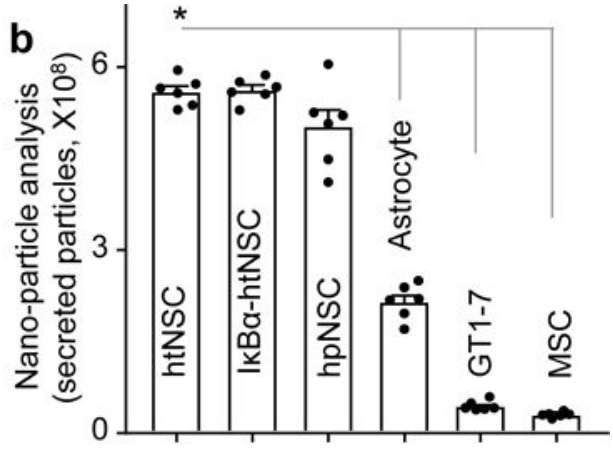

C
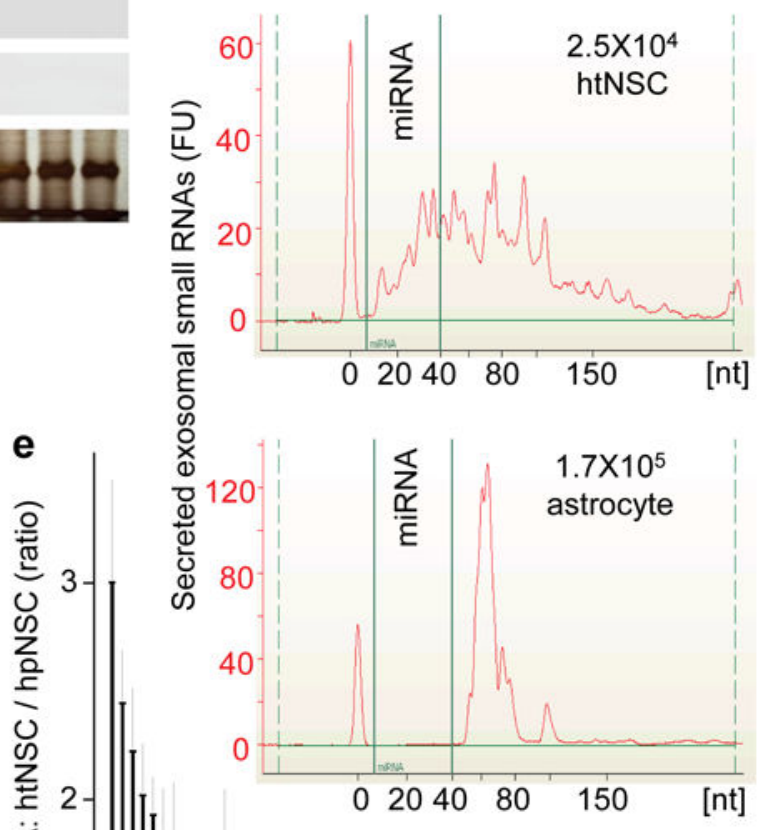

Figure 4. Exosomes and exosomal miRNAs secreted by hypothalamic NSC

Secretory exosomes isolated from the same number of indicated cells were assessed through Western blotting for indicated exosomal vs. non-exosomal markers (a) and analyses using Nano-particle tracking (b), small RNA bio-analyzer (c), and miRNA analysis via microarray chip (d) and qPCR (e). Results (e): secretory miRNA species identified to be abundantly released by hypothalamic NSC were compared to their release from hippocampal NSC (hpNSC). See details for (d) and (e) in Supplementary Data 1 and 2. $* p<10^{-4}$; one-way 
ANOVA with Tukey's test $(\mathbf{b}) ; n=3$ (a) or $n=6$ (b) independent samples per group or 2-3 independent experiments $(\mathbf{a}, \mathbf{c}, \mathbf{d}, \mathbf{e})$. Error bars reflect mean \pm s.e.m. 


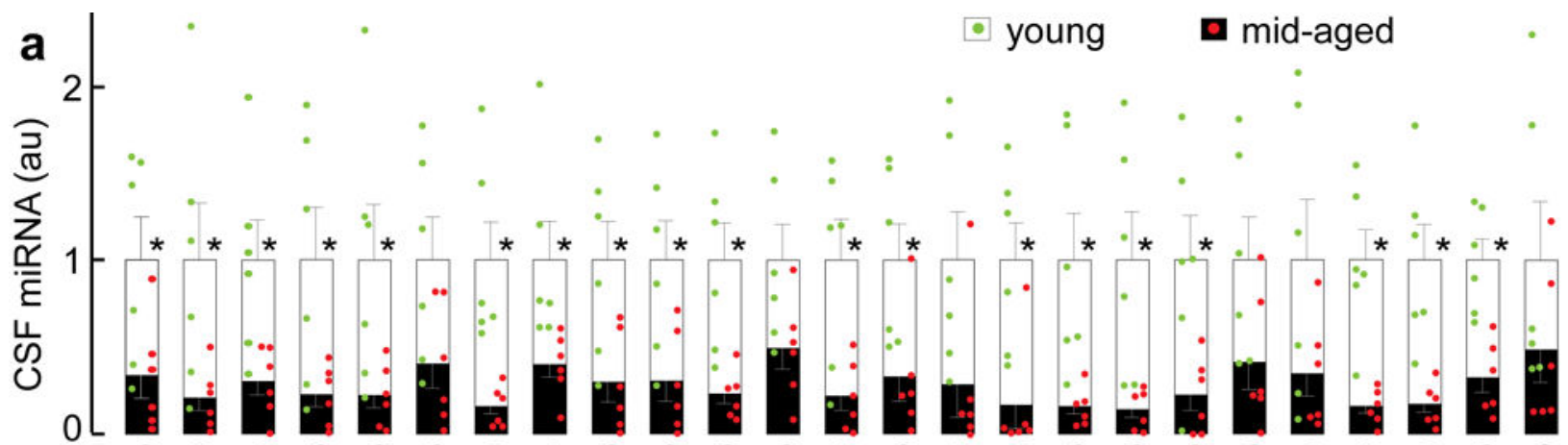

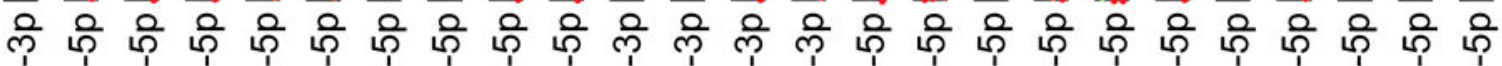

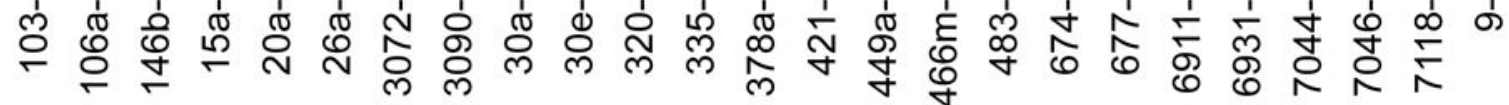

b

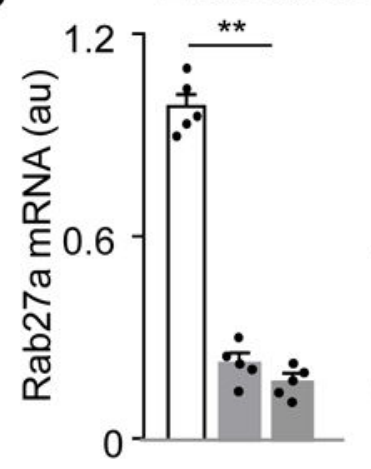

C
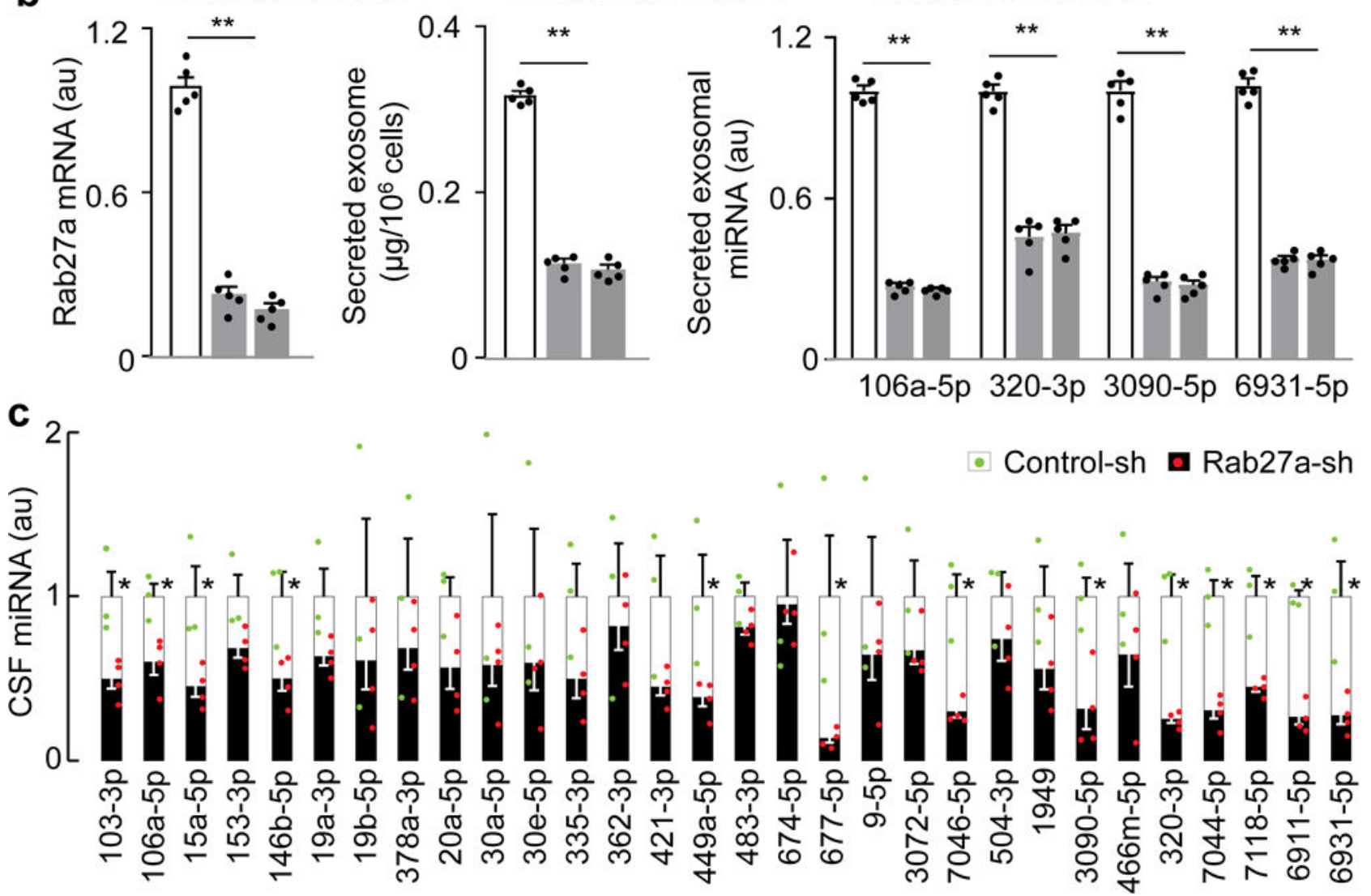

Figure 5. Contribution of hypothalamic NSC to exosomal miRNAs in the CSF

a, Exosomal miRNAs in the same volume of CSF from young vs. mid-aged C57BL/6 mice (each miRNA in young group normalized as 1). b, Cultured hypothalamic NSC were coinfected with Cre-dependent Rab27a shRNA (shRNA-1 or -2) vs. control scramble shRNA lentiviruses with Sox 2 promoter-driven Cre lentiviruses, and examined for Rab27a mRNA, secreted exosomes in the medium, and expression of indicated miRNAs in purified secreted exomsomes. c, C57BL/6 mice (4-month-old) were injected in hypothalamic 3V with Sox 2 promoter-driven Cre lentiviruses and Cre-dependent Rab27a shRNA vs. control scramble 
shRNA lentiviruses. CSF samples collected at 1 week post injection were analyzed for miRNAs. ${ }^{*} p<0.05, * * p<0.01$; two-tailed Student's t-test (a, c), one-way ANOVA with Tukey's test (b); $\mathrm{n}=6$ independent samples per group (a), $\mathrm{n}=3$ (Control-sh) or 4 (Rab27ash) independent samples per group (c), each sample was a pool of 3-4 mice (a, c), and $n=5$ independent samples per group (b); Error bars reflect mean \pm s.e.m. 

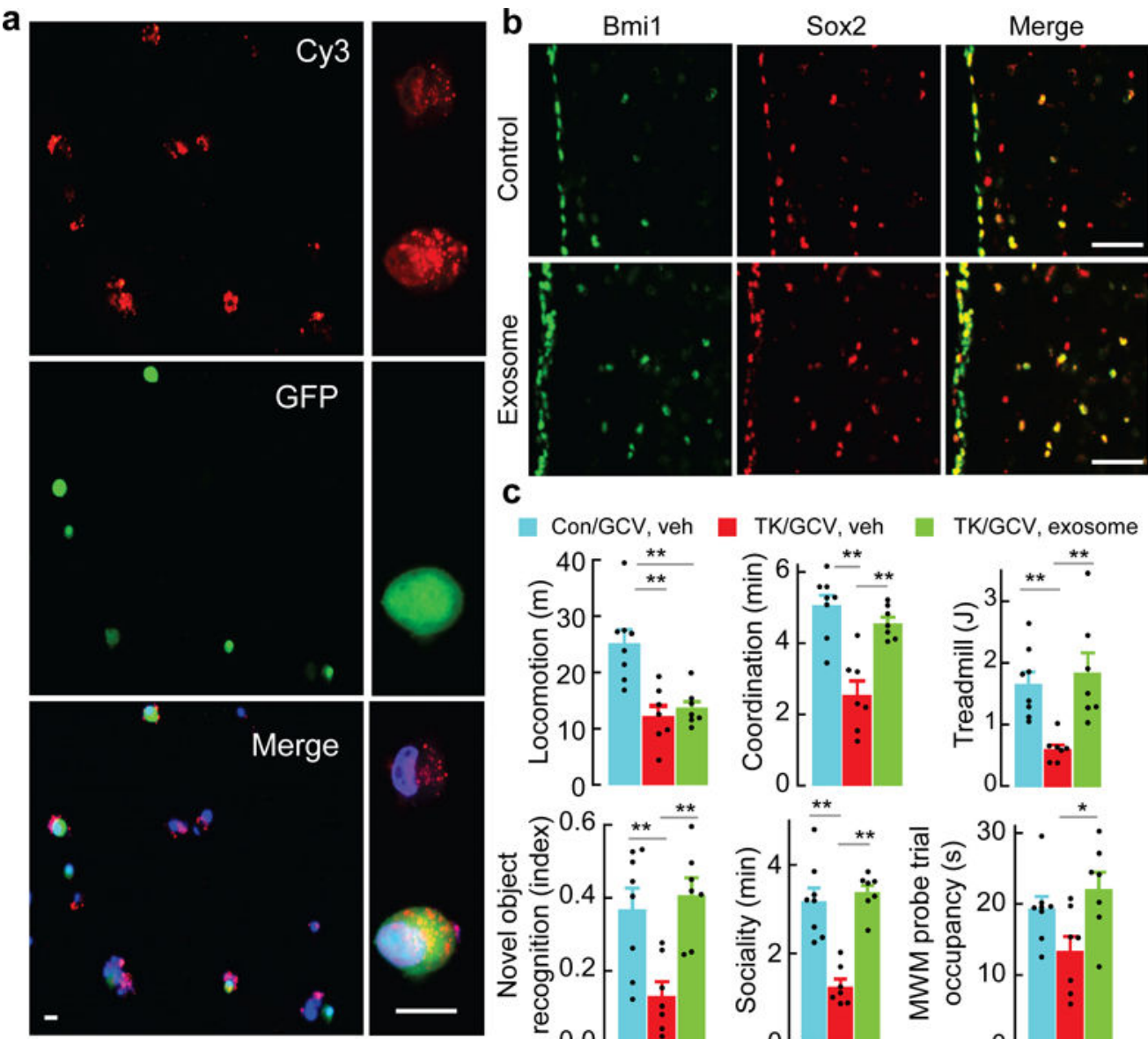

TK/GCV, veh TK/GCV, exosome
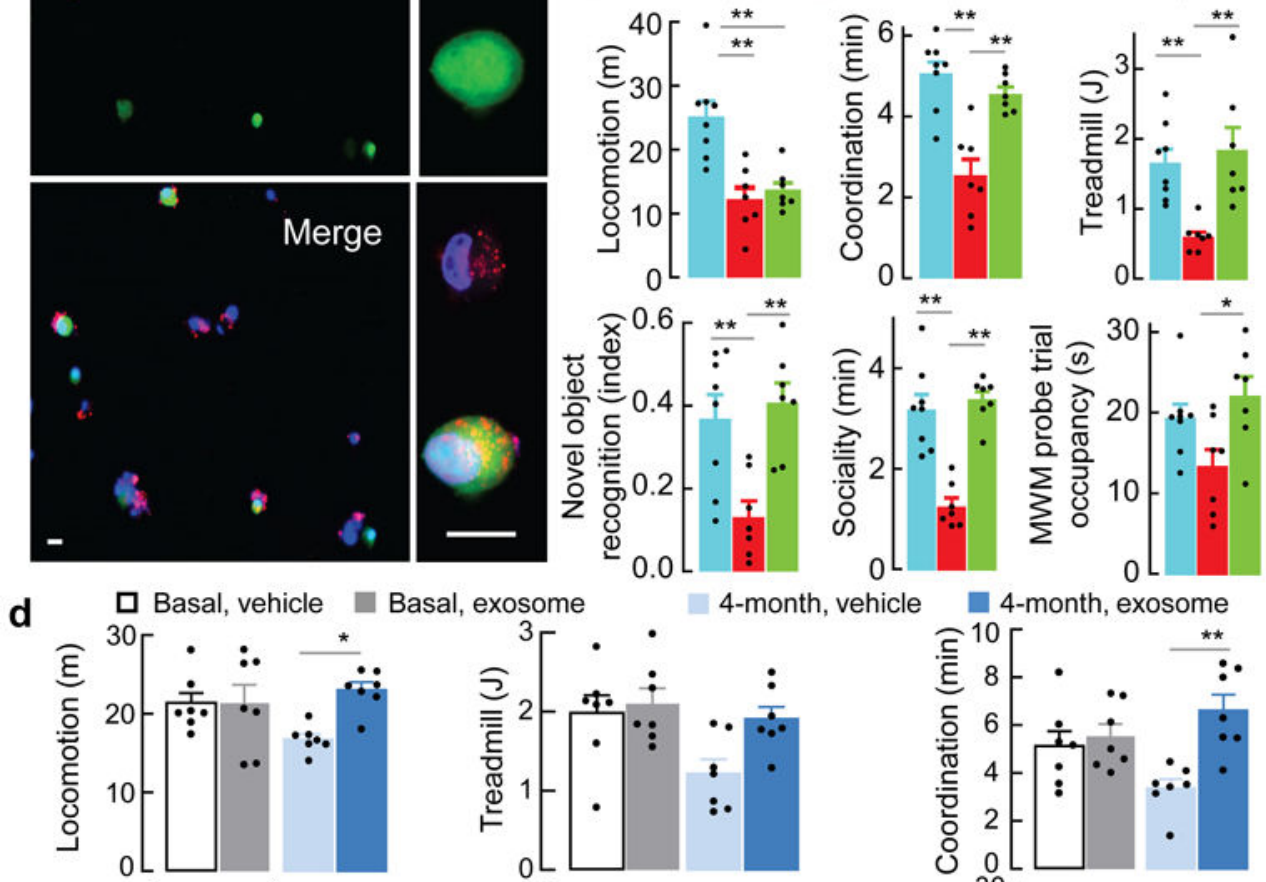

4-month, vehicle
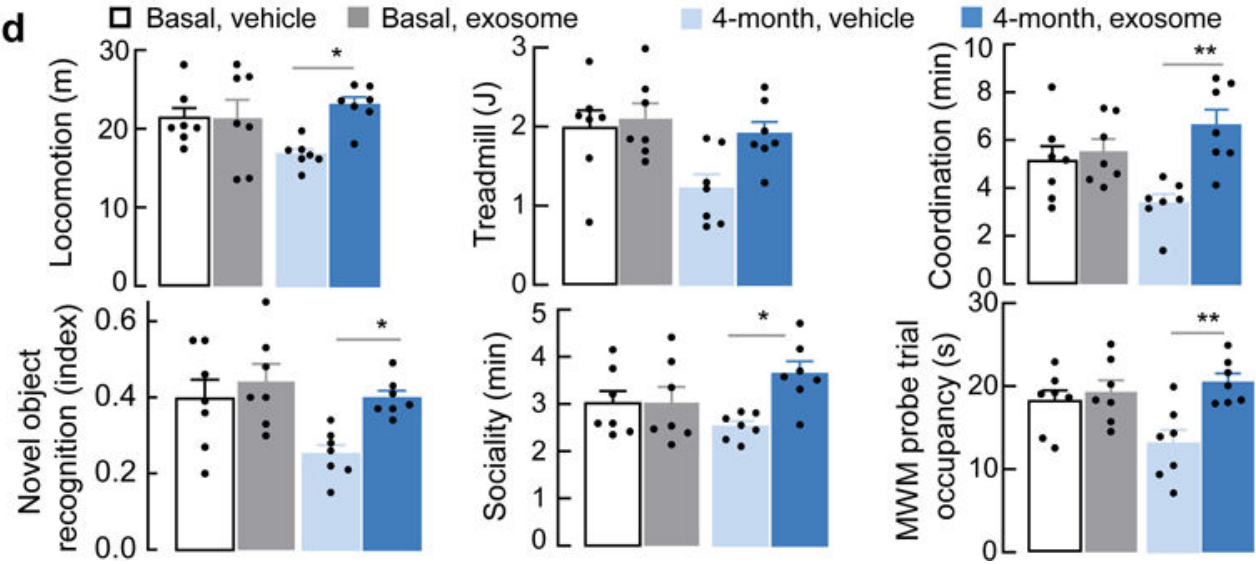

Figure 6. Slowdown of aging by treatment of hypothalamic NSC-derived exosomes

a, GFP-expressing htNSC transfected with Cy3-labeled miRNAs (106a-5p, 20a-5p and 466m-5p) were co-cultured with htNSC which did not contain GFP. Right panels: highmagnification images of representative GFP-positive and GFP-negative cells. Scale bar, 20 $\mu \mathrm{m}$. b, Mid-aged C57BL/6 mice were treated via hypothalamic 3V cannula with hypothalamic NSC-derived exosomes vs. vehicle, 3 times per week for 4 months, and examined for Bmi1 and Sox 2 in the MBH. Scale bar, $50 \mu \mathrm{m}$. c, Male C57BL/6 mice (15month-old) were bilaterally injected in the MBH with Bmil promoter-driven Hsv-TK (TK) 
or control (Con) lentiviruses, followed by GCV vs. vehicle (Veh) treatment, treated in hypothalamic $3 \mathrm{~V}$ with hypothalamic NSC-derived exosomes (2-3 times per week, 3 months), and examined for physiology. d, Male C57BL/6 mice (16-month-old) were treated in hypothalamic $3 \mathrm{~V}$ with exosomes vs. Veh (3 times per week, 4 months) and examined for physiology (d). Images represent 3 independent experiments $(\mathbf{a}, \mathbf{b}) . * p<0.05$, $* * p<0.01$; one-way ANOVA with Tukey's post-hoc test (c, d); $\mathrm{n}=8$ mice for Con/GCV-veh, and $\mathrm{n}=7$ mice for TK/GCV-veh and TK/GCV-exosome (c); $\mathrm{n}=7$ mice per group (d). Error bars reflect mean \pm s.e.m. 